\title{
A phase-field model for solute-assisted brittle fracture in elastic- plastic solids
}

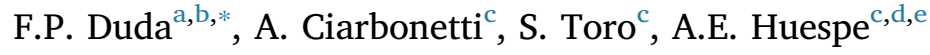 \\ a Programa de Engenharia Mecânica, COPPE, Universidade Federal do Rio de Janeiro, Cidade Universitária, Rio de Janeiro, CEP 21941-972, RJ, \\ Brazil \\ ${ }^{\mathbf{b}}$ Programa de Engenharia de Nanotecnologia, COPPE, Universidade Federal do Rio de Janeiro, Cidade Universitária, Rio de Janeiro, CEP 21941-972, \\ RJ, Brazil \\ ${ }^{\mathrm{c}}$ CIMEC-UNL-CONICET, Predio Conicet, Ruta Nac. $168 \mathrm{~s} / \mathrm{n}$ - Paraje El Pozo, 3000 Santa Fe, Argentina \\ d CIMNE - Centre Internacional de Mètodes Numèrics en Enginyeria, Spain \\ e T.S d'Enginyers de Camins, Canals i Ports, Technical University of Catalonia (Barcelona Tech) Campus Nord UPC, Mòdul C-1, C/ Jordi Girona 1-3, \\ 08034, Barcelona, Spain
}

\section{A R T I C L E I N F O}

\section{Keywords:}

Fracture

Elastoplasticity

Phase-field

Gradient damage mechanics

Hydrogen-assisted cracking

\begin{abstract}
A B S T R A C T
A phase-field theory of brittle fracture in elastoplastic solids hosting mobile interstitial solute species is developed in this paper. The theory, which is formulated within the framework of modern continuum mechanics, provides a systematic way to describe the interplay between solute migration and solid deformation and fracture. A specialization of the theory, which accounts for both solute-induced deformation and solute-assisted fracture as well as for their mutual effects on solute migration, is selected for numerical studies. Toward this end, a numerical model based on the finite-element method for spatial discretization and a splitting scheme with sub-stepping for the time integration is proposed. The model is applied to the study of hydrogenassisted crack propagation of high-strength steel specimens under sustained loads. The solutions obtained are compared with numerical and experimental results reported in the literature. It is shown that the proposed model has the capability to capture important features presented in the studied phenomenon.
\end{abstract}

\section{Introduction}

Multicomponent materials such as polymer gels, interstitial solid solutions and intercalation compounds have in common the nature of their constituents: they are composed of a deformable host solid of fixed composition and mobile guest species that can enter into, move through, and accumulate within the interstices of the host solid. These materials have definite shape inherited from the host component and variable composition since the amount of guest species within the host solid can change, and hence constitute a special class of solids of variable composition. Therefore, modeling the behavior of this type of solid requires a proper treatment of mechanical and chemical phenomena brought about by changes of shape and composition, including the incorporation of host-guest interactions. One example of such interaction is provided by the solid deformation caused by changes in the amount of a guest species, hereby called chemical deformation. In the case of elastic solids, such as reported by Larché and Cahn (1982), this interaction underlies the generation of stress by species migration and conversely species migration due to stress gradients. This

\footnotetext{
* Corresponding author. Programa de Engenharia Mecânica, COPPE, Universidade Federal do Rio de Janeiro, Cidade Universitária, Rio de Janeiro, CEP 21941-972, RJ, Brazil.

E-mail addresses: duda@mecanica.coppe.ufrj.br (F.P. Duda), angelodata@gmail.com (A. Ciarbonetti), storo@cimec.unl.edu.ar (S. Toro), ahuespe@intec.unl.edu.ar (A.E. Huespe).
} 
interplay between mechanics and chemistry gives rise to a rich variety of complex behaviors exhibited by many materials found in geology, polymer science and metallurgy (see Larché and Cahn, 1985, and references cited therein).

As described above, the study of multicomponent solids composed of elastic host solids and mobile guest species interacting via chemical deformation finds applications in many fields. Nevertheless, there are situations in which host solids can display inelastic behavior, such as plasticity and fracture, and additional host-guest interactions come into play by affecting the material properties of the host solid. For instance, guest hydrogen atoms can degrade plastic and fracture properties of host metals such as steels (see, for instance, Gangloff, 2003, and Hirth, 1980). This type of degradation, often called hydrogen embrittlement, can result in cracking and premature brittle failure of an otherwise safe material. Another very interesting example appears in the context of lithiation and delithiation of battery materials, wherein guest lithium ions can promote plasticity and fracture of a host silicon which otherwise exhibits elastic behavior (Zhao et al., 2011, Gao and Zhou, 2013, and Xu and Zhao, 2016).

Motivated by the foregoing remarks, we are concerned in this work with the formulation and numerical implementation of coupled theories for deformation, fracture and species migration in elastic-plastic solids. We restrict our attention to a single guest species, henceforth refereed to as solute. More specifically, the purpose of this work is threefold:

- to establish a framework to develop coupled theories of elastic-plastic deformation, fracture and solute migration in solids;

- to specialize the general framework to obtain a particular theory that incorporates some of the most essential features that arise in the context of hydrogen embrittlement;

- to propose a numerical technique based on the finite element method for spatial discretization and a splitting scheme with substepping for the time integration of the evolution equation system governing hydrogen embrittlement in high-strength steel under sustained loads.

Following the framework of modern continuum mechanics (Gurtin et al., 2010), we develop a general theory of elastic-plastic deformation, fracture and solute migration in solids by proceeding in four steps, describing the kinematics, basic balances, energy imbalance and constitutive theory. Accordingly, we enrich the standard kinematical description of a solid body by adding three additional scalar fields to represent plasticity, fracture and solute migration. Then, after choosing the forms of the internal and external power expenditures, we derive all basic balances of the theory by using the method of virtual power and postulate the energy imbalance. Finally, we introduced a constitutive theory consistent with the energy imbalance that can incorporate different solidsolute interactions. The resulting theory extends the one presented by Duda et al. (2015) by including gradient plasticity and solute migration. The approach to gradient plasticity is similar to the one adopted by Gurtin and Anand (2009) in that the accumulated plastic strain is viewed as an independent kinematic descriptor (see also Duda and Souza, 2002, for related developments), whereas the treatment of solute migration follows Duda et al. (2010) by considering the solute chemical potential as a generalized velocity. As in da Silva et al. (2013), a scalar kinematical descriptor, referred to as damage variable or phase field, is introduced to describe fracture. The foregoing works also use the framework of continuum mechanics.

With a view towards application to hydrogen-assisted cracking (HAC) problems, one particular realization of the general theory is singled out for detailed studies. This particular theory accounts for solid-solute interactions brought about by the local deformation and fracture-energy degradation of the host solid induced by the presence of the guest solute. On combining the mentioned constitutive information with the balances of the general theory, we arrive at a system of coupled equations governing the evolution of the displacement, hydrogen concentration, and phase field. This particular theory can be seen as an extension of those presented in (Duda et al., 2007) and (Duda et al., 2009) to account for plasticity.

Finally, we propose a strategy for the numerical implementation of governing equations using the finite-element method for the spatial discretization and a staggered scheme for the time integration. This strategy follows the basic guidelines proposed by the authors in (Duda et al., 2015). We remark that the works of Miehe and coauthors, about numerical techniques of phase field models for crack propagation problems in elastic and plastic solids, have been a source of motivation for the present numerical implementation, see for example Miehe et al. (2010) and Miehe et al. (2016) and references cited therein. Also, it is worth mentioning the recent phase-field approaches to crack growth in the presence of solute diffusion reported by Klinsmann et al. (2016) and Wu and De Lorenzis (2016).

Several tests of center notched panels in the presence of an aggressive environment are simulated to illustrate the use of the implemented theory. The panels are constituted of different high-strength steels and are subjected to sustained loads. These loads are lower than the safe design ones in non-corrosive environments. The assumed embrittlement mechanism to induce the fracture initiation is the degradation, or reduction, of the cohesive strength and fracture toughness due to a build-up of hydrogen in the material. This mechanism for explaining the highly complex hydrogen-assisted cracking phenomenon goes back to the original models of Troiano (2016) and Oriani and Josephic (1974), then followed by a number of authors, Gerberich and Chen (1975), Unger (1989), Dadfarnia et al. (2014), etc.

The numerical simulation of these panels takes into account the following coupled phenomena: i) plastic strains in regions close to the notch, ii) hydrogen uptake from the environment through the notch and crack flanks and the related stress-assisted bulk diffusion, iii) metal embrittlement due to hydrogen, as well as, iv) crack initiation and propagation through the specimen. Interaction between these phenomena are particularly analyzed in the numerical solutions. The objectives here pursued with these simulations are:

- to evaluate the role of different parameters characterizing the present methodology in order to reproduce distinctive experimental facts already published in the literature; and

- to compare the numerical solutions provided by the phase field model against similar previously reported results which have been 
obtained using alternative computational approaches.

The remainder of this paper is organized as follows. The theoretical framework is developed in Section 2. A particular specialization of the framework is presented in Section 3. An illustrative example is presented in Section 4. Finally, some concluding remarks are provided in Section 5. Details of the numerical implementation of this specialization are given in Appendix I.

\section{General theory}

In this paper, we consider a material body $\mathcal{B}$ composed of two physical sub-structures: an elastic-plastic host solid of fixed composition that can undergo fracture and a guest solute that is free to move through the interstices of the host solid. Such a body is the seat of the following, possibly interdependent, phenomena: elastic-plastic deformation and fracture experienced by the host solid and solute migration. Our task here is to formulate a general continuum theory for the interaction between these phenomena. We will restrict attention to small-strain and isothermal conditions.

We begin the formulation by enriching the classical kinematical description of a solid in terms of the displacement field, $\mathbf{u}$, by adding three scalar fields to represent the kinematics of plasticity, fracture and solute migration: the accumulated plastic strain field $e$; the damage or phase field $\varphi$, which ranges smoothly between $\varphi=0$ (unfractured solid) and $\varphi=1$ (fractured solid); the chemical displacement field $\alpha$, the time derivative of which is the chemical potential of the solute in the solid. Then, we introduce four balanced force systems conjugated to the foregoing kinematical descriptors by choosing the forms of the internal and external power expenditures and invoking the method of virtual power. After splitting the external forces into inertial and non-inertial, or applied, parts, we also introduce a mechanical version of the second law of thermodynamics that involves the total energy and the power of all applied forces. With appropriate prescriptions for the inertial forces, we arrive at the basic balances of the theory, namely the linear momentum balance, solute content balance, and the two force balances associated with plasticity and fracture, and the free-energy imbalance that is used as a guide for developing a constitutive theory consistent with thermodynamics.

The constitutive theory is developed by assuming the additive decomposition of the solid strain into elastic, plastic and chemical parts, with the plastic and chemical strain rates proportional to the rates of accumulated plastic strain and solute density. Then, guided by the free-energy imbalance, we consider that the free energy density, chemical potential, stress tensor, migrative flux vector, as well as the stress vectors and internal forces associated with plasticity and fracture, are related to the elastic strain, solute density, accumulated plastic strain, and phase field. The requirement of thermodynamic consistency implies that the constitutive equations are defined in terms of the response functions for free energy density, migrative flux vector, and dissipative parts of the internal forces introduced to describe plasticity and fracture.

In particular, we show that the migrative flux is proportional to the gradient of the solute chemical potential, which by its turn is given by the sum of the partial derivative of the free energy response with respect to the solute density and a stress-dependent term that appears as a reciprocal effect of the solute-induced strain. Therefore, solute migration driven by stress, elastic strain, accumulated plastic strain and damage appears as reciprocal effects of solute-induced deformation and solute-induced modification of the elastic, plastic and fracture properties appearing in the free energy response. Additional solid-solute interactions can be implemented through the response functions for dissipative parts of the internal forces introduced to describe plasticity and fracture.

Our treatment of the chemical potential allow us to treat all phenomena considered in the paper on the same footing in the following sense: all basic balances, including the solute content force balance, are derived by using the principle of virtual power; all energy-inflow contributions appearing in the energy imbalance are described as power expenditures. The mentioned approach was inspired by the virtual-power treatment of thermomechanics pioneered in Podio-Guidugli (2009), where the temperature, like the chemical potential in our case, was treated as a generalized velocity. Alternatively, one could follow an approach in which all basic balances are postulated globally for material regions and introduce the chemical potential notion to characterize the energy inflow by solute transport, see for instance Gurtin et al. (2010).

\subsection{Kinematics}

We now discuss the selection of the independent fields that will be used to describe the kinematical structure of $\mathcal{B}$, wherein the classical framework introduced to represent deformation in solids will be enriched to incorporate the generalized kinematics of plasticity, fracture and solute migration. In this regard, we view the solid solution $\mathcal{B}$ as a solid with microstructure (Capriz, 1989). We henceforth consider $\mathcal{B}$ identified with the region it occupies in a fixed reference configuration.

The standard kinematics of $\mathcal{B}$ can be characterized by a vector field $\mathbf{u}$ that ascribes to each material point $\mathbf{x}$ in $\mathcal{B}$ and time $t$ the corresponding displacement vector $\mathbf{u}(\mathbf{x}, t)$ at time $t$. In particular, the local deformation of $\mathcal{B}$ is determined by the infinitesimal strain tensor field $\mathbf{E}$ defined by:

$$
\mathbf{E}=\frac{\nabla \mathbf{u}+\nabla \mathbf{u}^{\top}}{2}
$$

Hereafter, the tensor field $\nabla \mathbf{w}$ denotes the gradient of a vector field $\mathbf{w}$ and $\mathbf{A}^{\top}$ the transpose of a tensor $\mathbf{A}$.

To motivate our choice for the kinematical field to represent plasticity, we anticipate the decomposition of the strain $\mathbf{E}$ into its elastic $\mathbf{E}_{\mathrm{e}}$, plastic $\mathbf{E}_{\mathrm{p}}$ and solute-induced, or chemical, $\mathbf{E}_{\mathrm{c}}$ parts. The plastic strain rate $\dot{\mathbf{E}}_{\mathrm{p}}$ is a non-null tensor whenever plastic flow takes place, which suggests that $\left|\dot{\mathbf{E}}_{\mathrm{p}}\right|$ can be used as a measure of the plastic flow intensity. This lead us to stipulate that the kinematics of plasticity can be described by the scalar field $e$, with $\dot{e}=\left|\dot{\mathbf{E}}_{\mathrm{p}}\right|$. Notice that, by definition, $e$ assigns to each material point $\mathbf{x}$ in $\mathcal{B}$ and 
time $t$ the scalar

$$
e(\mathbf{x}, t)=\int_{0}^{t}\left|\dot{\mathbf{E}}_{\mathrm{p}}(\mathbf{x}, \omega)\right| d \omega .
$$

For simplicity, we assume that $\mathcal{B}$ is free of plastic strain at $t=0$ and refer to the field $e$ as the accumulated plastic strain.

We picture the fracture of $\mathcal{B}$ as the ultimate result of bond-breakage processes experienced by the host solid and consider their kinematics described through a single scalar field $\varphi$ that assigns to each material point $\mathbf{x}$ in $\mathcal{B}$ the corresponding level of material degradation at time $t, \varphi(\mathbf{x}, t)$. It takes values on the interval $[0,1]$ and if $\varphi=0$ at a point, then the material is pristine at that point. If $\varphi=1$ at some other point, then the material is fractured at that point. Accordingly, cracks can be viewed as small regions in which the variation of $\varphi$ between zero and one are confined. We refer to the field $\varphi$ as the damage, or phase, field.

We recall that $\mathcal{B}$ changes its composition due to solute migration and consider that the tendency to this change can be described by a single scalar field $\mu$ henceforth denoted as solute chemical potential. We refer the reader to Baierlein (2001) and Job and Herrmann (2006) for a similar definition of chemical potential. Then, following Duda et al. (2010), we assume that the kinematics of composition changes is determined by the scalar field $\alpha$ defined by the relation to each material point $\mathbf{x}$ in $\mathcal{B}$ and time $t$, which implies that $\dot{\alpha}=\mu$,

$$
\alpha(\mathbf{x}, t)=\int_{0}^{t} \mu(\mathbf{x}, \omega) d \omega .
$$

To summarize, we assume that $\mathcal{B}$, from the point of view of kinematics, is described through the following fields: displacement $\mathbf{u}$, accumulated plastic strain $e$, damage $\varphi$ and chemical displacement $\alpha$. The corresponding realizable velocities are denoted by $\mathbf{v}=\dot{\mathbf{u}}, \dot{e}$, $\dot{\varphi}$, and $\mu=\dot{\alpha}$.

For later reference, we introduce the scalar fields $\rho$ and $c$ to represent the contents of the solid and solute components of $\mathcal{B}$. They assign to each material point $\mathbf{x}$ in $\mathcal{B}$ and time $t$ the corresponding mass density $\rho(\mathbf{x}, t)$ and molar density $c(\mathbf{x}, t)$, measured in mass of the host solid and moles of solute atoms per unit reference volume, at time $t$. Notice that $\dot{\rho}=0$ since, by definition, the amount of host solid is conserved. This implies that the composition of $\mathcal{B}$ is fully specified by the field $c$.

\subsection{Basic balances}

We now introduce the basic balances of the theory, namely the linear momentum balance, solute content balance and two additional force balances to account for plasticity and fracture. Rather than being postulated, these balances are derived by invoking the principle of virtual power. The version of the principle of the virtual power adopted here is stated for arbitrary parts $\mathscr{P}$ of $\mathcal{B}$.

\subsubsection{Virtual powers}

For a fixed time $t$, let $\mathscr{V}$ denote the set of all smooth virtual velocity fields $(\widetilde{\mathbf{u}}, \tilde{e}, \widetilde{\varphi}, \widetilde{\alpha})$ on $\mathcal{B}$, a set which includes the realizable velocities $(\dot{u}, \dot{e}, \dot{\varphi}, \mu)$. The method of virtual power (e.g. Germain, 1973) predicates that the dynamics of $\mathcal{B}$ is specified in terms of a pair of linear, bounded, and continuous functionals on $\mathscr{V}$, referred to as the internal and external virtual power expenditures, defined for arbitrary parts $\mathscr{P}$ of $\mathcal{B}$. Here, we stipulate that the internal virtual power expenditure is given by

$$
\mathscr{W}_{i}(\mathscr{P} ; \tilde{\mathbf{u}}, \tilde{e}, \tilde{\varphi}, \widetilde{\alpha}):=\int_{\mathscr{P}}(\mathbf{S} \cdot \nabla \widetilde{\mathbf{u}}-\mathbf{h} \cdot \nabla \widetilde{\alpha}+\tau \cdot \nabla \tilde{e}+\xi \cdot \nabla \tilde{\varphi}+\gamma \tilde{e}+\pi \widetilde{\varphi}) \mathrm{d} V,
$$

where $\mathbf{S}$ is the stress tensor, $\mathbf{h}$ the solute flux vector, $\tau$ and $\gamma$ the microstress vector and internal body force associated to plasticity, and $\xi$ and $\pi$ the microstress vector and internal body force associated to decohesion. We also stipulate that the external virtual power expenditure is expressed as

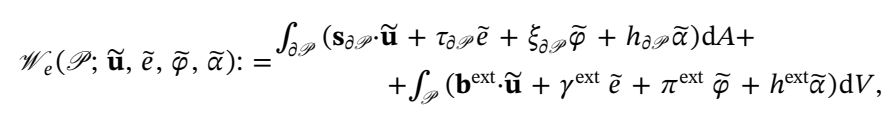

$\mathbf{s}_{\partial \mathscr{P}}, \tau_{\partial \mathscr{P}}, \xi_{\partial \mathscr{P}}, h_{\partial \mathscr{P}}$ represent contact interactions and $\mathbf{b}^{\mathrm{ext}}, \gamma^{\mathrm{ext}}, \pi^{\mathrm{ext}}$, and $h^{\mathrm{ext}}$ interactions at a distance, that is, they account for interactions with all bodies external to $\mathcal{B}$.

The interactions at distance can be split into non-inertial, or applied, and inertial parts. The non-inertial parts of $\mathbf{b}^{\mathrm{ext}}, \gamma^{\mathrm{ext}}, \pi^{\mathrm{ext}}$, and $h^{\text {ext }}$ account for interactions of bodies accessible to observation, whereas the inertial parts of $\mathbf{b}^{\text {ext }}, \gamma^{\text {ext }}, \pi^{\text {ext }}$, and $h^{\text {ext }}$ account for interactions with bodies in the remaining universe. See the discussion provided by Truesdell (1992). Here, we assume that $\gamma^{\text {ext }}$ and $\pi^{\text {ext }}$ are purely non-inertial and adopt the following decompositions of $\mathbf{b}^{\text {ext }}$ and $h^{\text {ext }}$ :

$$
\mathbf{b}^{\text {ext }}=\mathbf{b}-\dot{\mathbf{p}} \text { and } h^{\mathrm{ext}}=h^{\mathrm{ni}}-\dot{c},
$$

where $\mathbf{b}$ and $h^{\mathrm{ni}}$ are the non-inertial parts of $\mathbf{b}^{\text {ext }}$ and $h^{\text {ext }},-\dot{\mathbf{p}}$ and $-\dot{c}$ are the corresponding inertial parts. The fields $\mathbf{p}$ and $c$ represent, respectively, the linear momentum and solute number densities. On substituting (6) into (5), it follows that the external power $\mathscr{W}_{e}$ admits the decomposition into a "non-inertial" part $\mathscr{W}_{e}^{\text {ni }}$ and an "inertial" part $\mathscr{W}_{e}^{\text {in }}$, with

$$
\mathscr{W}_{e}=\mathscr{W}_{e}^{\mathrm{ni}}+\mathscr{W}_{e}^{\mathrm{in}}
$$




$$
\mathscr{W}_{e}^{\mathrm{ni}}(\mathscr{P} ; \widetilde{\mathbf{u}}, \tilde{e}, \tilde{\varphi}, \tilde{\alpha}):=\begin{aligned}
& \int_{\mathscr{P}}\left(\mathbf{b} \cdot \widetilde{\mathbf{u}}+\gamma^{\mathrm{ext}} \tilde{e}+\pi^{\mathrm{ext}} \tilde{\varphi}+h^{\mathrm{ni}} \tilde{\alpha}\right) \mathrm{d} V+ \\
& +\int_{\partial \mathscr{P}}\left(\mathbf{s}_{\partial \mathscr{P}} \cdot \widetilde{\mathbf{u}}+\tau_{\partial \mathscr{P}} \tilde{e}+\xi_{\partial \mathscr{P}} \tilde{\varphi}+h_{\partial \mathscr{P}} \tilde{\alpha}\right) \mathrm{d} A
\end{aligned}
$$

and

$$
\mathscr{W}_{e}^{\mathrm{in}}(\mathscr{P} ; \widetilde{\mathbf{u}}, \tilde{e}, \widetilde{\varphi}, \widetilde{\alpha}):=-\int_{\mathscr{P}}(\dot{\mathbf{p}} \cdot \widetilde{\mathbf{u}}+\dot{c} \widetilde{\alpha}) \mathrm{d} V .
$$

The principle of virtual power, which is assumed to hold at each fixed time, states that, for any part $\mathscr{P}$ of $\mathcal{B}$,

$$
\mathscr{W}_{i}(\mathscr{P} ; \tilde{\mathbf{u}}, \tilde{e}, \widetilde{\varphi}, \widetilde{\alpha})=\mathscr{W}_{e}(\mathscr{P} ; \tilde{\mathbf{u}}, \tilde{e}, \widetilde{\varphi}, \widetilde{\alpha})
$$

for every $(\widetilde{\mathbf{u}}, \tilde{e}, \widetilde{\varphi}, \widetilde{\alpha})$ in $\mathscr{V}$.

This principle can be used to get the basic balances localized to material points in $\mathcal{B}$ and on its boundary $\partial \mathcal{B}$.

\subsubsection{Basic balances in local form}

We now consider (10) applied to the entire body $\mathcal{B}$. Then, after using the fundamental lemma of calculus of variations, we arrive at the following expressions representing the basic balances in local form:

- Linear momentum balance

$$
\operatorname{Div} \mathbf{S}+\mathbf{b}=\dot{\mathbf{p}} \quad \text { on } \mathcal{B} \text { and } \quad \mathbf{S n}=\mathbf{s}_{\partial \mathcal{B}} \quad \text { on } \partial \mathcal{B},
$$

where $\mathbf{S}$ is symmetric thanks to the frame-indifference of the internal power;

- Solute content balance

$$
-\operatorname{Div} \mathbf{h}+h^{\mathrm{ni}}=\dot{c} \quad \text { on } \mathcal{B} \quad \text { and } \quad-\mathbf{h} \cdot \mathbf{n}=h_{\partial \mathcal{B}} \quad \text { on } \partial \mathcal{B} .
$$

- Phase-field microforce balance

$$
\operatorname{Div} \boldsymbol{\xi}-\pi+\pi^{\mathrm{ext}}=0 \quad \text { on } \mathcal{B} \quad \text { and } \quad \boldsymbol{\xi} \cdot \mathbf{n}=\xi_{\partial \mathcal{B}} \quad \text { on } \partial \mathcal{B} ;
$$

- Plastic-strain microforce balance

$$
\operatorname{Div} \tau-\gamma+\gamma^{\mathrm{ext}}=0 \quad \text { on } \mathcal{B} \quad \text { and } \quad \boldsymbol{\tau} \cdot \mathbf{n}=\tau_{\partial \mathcal{B}} \quad \text { on } \partial \mathcal{B}
$$

where $\mathbf{n}$ is the exterior unit vector normal to $\partial \mathcal{B}$.

\subsection{Energy imbalance}

In addition to the force balances introduced before, we impose the first and second laws of thermodynamics via an energy imbalance. This imbalance requires that the rate of change of the free energy of any $\mathscr{P}$ does not exceed the inflow of energy to $\mathscr{P}$ due to the power expended on $\mathscr{P}$ by external agencies.

Then, by assuming that the momentum density $\mathbf{p}$ admits the classical representation $\mathbf{p}=\rho \dot{\mathbf{u}}$, where $\rho$ is the mass density of the host solid which, since the host solid is conserved, satisfies the condition $\dot{\rho}=0$. And after introducing the standard notion of specific kinetic energy $\kappa=\rho|\dot{\mathbf{u}}|^{2} / 2$, as well as the free energy density per unit reference volume, $\psi$, it follows the law of energy imbalance for $\mathscr{P}$ which reads

$$
\frac{d}{d t} \int_{\mathscr{P}}(\psi+\kappa) \mathrm{d} V \leq \mathscr{W}_{e}^{\mathrm{ni}}(\mathscr{P} ; \dot{\mathbf{u}}, \dot{e}, \dot{\varphi}, \mu)
$$

Bearing in mind that $\mathscr{P}$ is arbitrary, making use of (7), (8), (10), and recalling that $\mathbf{S}$ is symmetric, we find that the integral statement (15) of energy imbalance has the equivalent point-wise version

$$
\dot{\psi}-\mu \dot{c}-\mathbf{S} \cdot \dot{\mathbf{E}}-\tau \cdot \nabla \dot{e}-\gamma \dot{e}-\xi \cdot \nabla \dot{\varphi}-\pi \dot{\varphi}+\mathbf{h} \cdot \nabla \mu \leq 0
$$

\subsection{Constitutive theory}

We now develop a constitutive theory that allows for the incorporation of various couplings between elastic-plastic deformation, fracture and solute migration in a solid solution $\mathcal{B}$.

Our theory is based on the additive decomposition of the strain $\mathbf{E}$ into elastic $\mathbf{E}_{\mathrm{e}}$, plastic $\mathbf{E}_{\mathrm{p}}$ and solute-induced $\mathbf{E}_{\mathrm{c}}$ parts:

$$
\mathbf{E}=\mathbf{E}_{\mathrm{e}}+\mathbf{E}_{\mathrm{p}}+\mathbf{E}_{\mathrm{c}}
$$

Here, we restrict our attention to metal plasticity and assume that the plastic strain $\mathbf{E}_{\mathrm{p}}$ is deviatoric, that is, $\operatorname{tr} \mathbf{E}_{\mathrm{p}}=0$. This implies that the plastic strain rate $\dot{\mathbf{E}}_{\mathrm{p}}$ admits the representation 


$$
\dot{\mathbf{E}}_{\mathrm{p}}=\dot{e} \mathbf{N}
$$

with the plastic-flow direction tensor $\mathbf{N}$ satisfying the conditions

$$
|\mathbf{N}|=1, \quad \operatorname{tr}(\mathbf{N})=0 .
$$

As for the solute-induced strain $\mathbf{E}_{\mathrm{c}}$, which represents the local deformation caused by changes in composition (Larché and Voorhees, 1996), we assume that it is purely dilatational and given by

$$
\mathbf{E}_{c}=\frac{v}{3}\left(c-c_{0}\right) \mathbf{I}
$$

where $v>0$ represents the increase of the solid solution volume per mole of solute atoms and $c_{0}$ is the solute molar density in the reference configuration used to measure strain. We also assume that $\mathcal{B}$ is subjected to the internal constraints which state that microstructural changes leading to plasticity and fracture are irreversible:

$$
\dot{e} \geq 0, \quad \dot{\varphi} \geq 0 .
$$

Taking into account the assumptions represented by equations (17)-(20), we rewrite (16) as

$$
\dot{\psi}-\bar{\mu} \dot{c}-\mathbf{S} \cdot \dot{\mathbf{E}}_{\mathrm{e}}-\tau \cdot \nabla \dot{e}-\bar{\gamma} \dot{e}-\xi \cdot \nabla \dot{\varphi}-\pi \dot{\varphi}+\mathbf{h} \cdot \nabla \mu \leq 0
$$

where

$$
\bar{\mu}=\mu+v \sigma_{\mathrm{m}}, \quad \bar{\gamma}=\gamma+\tau,
$$

$\sigma_{\mathrm{m}}=\mathbf{S} \cdot \mathbf{I} / 3$ is the hydrostatic stress and $\tau=\mathbf{S}_{0} \cdot \mathbf{N}$ is the resolved stress obtained by projecting $\mathbf{S}_{0}$, the deviatoric part of $\mathbf{S}$, along the plastic flow direction.

Guided by the structures of the internal constraints (21) and the dissipation inequality (22), we now establish the quantities to which constitutive relations will be formulated. We begin by recalling that the standard practice of dealing with internal constraints (e.g. Capriz, 1989) dictates that: $\mathbf{S}, \tau, \xi, \pi, \bar{\gamma}, \bar{\mu}$ and $\mathbf{h}$ are sums of two components, one called active and the other called reactive; the reactive contributions expend no power and constitutive relations can be assigned only for the active components. Then, after some calculations involving the arguments presented in da Silva et al. (2013) and Duda et al. (2015), we conclude that whereas the reactive parts of $\mathbf{S}, \tau, \xi, \bar{\mu}$ and $\mathbf{h}$ must vanish, the reactive parts of $\pi$ and $\bar{\gamma}$ vanish when $\dot{\varphi} \neq 0$ and $\dot{e} \neq 0$ but are otherwise undetermined. Therefore, we have to introduce constitutive relations for $\psi, \mathbf{S}, \tau, \xi, \bar{\mu}$ and $\mathbf{h}$, and for the active parts of $\pi$ and $\bar{\gamma}$, namely $\pi_{\mathrm{a}}$ and $\bar{\gamma}_{\mathrm{a}}$.

For later reference, we emphasize that $\pi$ and $\bar{\gamma}$ admit the representation

$$
\bar{\gamma}=\bar{\gamma}_{\mathrm{a}}+\bar{\gamma}_{\mathrm{r}}, \quad \pi=\pi_{\mathrm{a}}+\pi_{\mathrm{r}}
$$

where

$$
\bar{\gamma}_{\mathrm{r}}=\left\{\begin{array}{cl}
\text { undetermined, } & \text { if } \dot{e}=0, \\
0, & \text { if } \dot{e}>0,
\end{array}\right.
$$

and

$$
\pi_{\mathrm{r}}=\left\{\begin{array}{cl}
\text { undetermined, } & \text { if } \dot{\varphi}=0 \\
0, & \text { if } \dot{\varphi}>0
\end{array}\right.
$$

\subsubsection{Constitutive response functions}

As discussed before, we have to introduce constitutive relations for $\psi, \mathbf{S}, \tau, \boldsymbol{\xi}, \bar{\mu}, \mathbf{h}, \pi_{\mathrm{a}}$ and $\overline{\gamma_{\mathrm{a}}}$. Guided by the structure of the inequality (22), we allow for possible constitutive dependencies of these quantities on the list $(\varsigma, \dot{e}, \dot{\varphi}, \nabla \mu)$, where

$$
\varsigma=\left(\mathbf{E}_{\mathrm{e}}, e, \varphi, c, \nabla e, \nabla \varphi\right)
$$

Then, granted the availability of external forces needed to ensure the satisfaction of the local balances $(11)_{1},(12)_{1},(13)_{1}$ and $(14)_{1}$, a straightforward application of an argument first employed by Coleman and Noll (1963) shows that $\psi$ must be independent of $\dot{e}, \dot{\varphi}$ and $\nabla \mu$, that is,

$$
\psi=\psi(\varsigma)
$$

that $\mathbf{S}, \tau, \boldsymbol{\xi}$ and $\bar{\mu}$ must be given by equations of state

$$
\mathbf{S}=\frac{\partial \psi(\varsigma)}{\partial \mathbf{E}_{\mathrm{e}}}, \quad \tau=\frac{\partial \psi(\varsigma)}{\partial \nabla e}, \quad \xi=\frac{\partial \psi(\varsigma)}{\partial \nabla \varphi}, \quad \bar{\mu}=\frac{\partial \psi(\varsigma)}{\partial c},
$$

and that $\bar{\gamma}_{\mathrm{a}}$ and $\pi_{\mathrm{a}}$ must obey the residual dissipation inequality

$$
\begin{aligned}
&\left(\bar{\gamma}_{\mathrm{a}}(\varsigma, \dot{e}, \dot{\varphi}, \nabla \mu)-\frac{\partial \psi(\varsigma)}{\partial e}\right) \dot{e}+\left(\pi_{\mathrm{a}}(\varsigma, \dot{e}, \dot{\varphi}, \nabla \mu)-\frac{\partial \psi(\varsigma)}{\partial \varphi}\right) \dot{\varphi}- \\
&-\mathbf{h}(\varsigma, \dot{e}, \dot{\varphi}, \nabla \mu) \cdot \nabla \mu \geq 0
\end{aligned}
$$


for all $(\varsigma, \dot{e}, \dot{\varphi}, \nabla \mu)$ compatible with (21).

Instead of determining the most general constitutive response functions $\bar{\gamma}_{\mathrm{a}}, \pi_{\mathrm{a}}$ and $\mathbf{h}$ satisfying (30), we suppose that $\bar{\gamma}_{\mathrm{a}}$ is independent of $\dot{\varphi}$ and $\nabla \mu, \pi_{\mathrm{a}}$ independent of $\dot{e}$ and $\nabla \mu$, and $\mathbf{h}$ independent of $\dot{e}$ and $\dot{\varphi}$. Thus, we restrict attention to solutions for which the dissipative mechanisms associated with plasticity, fracture, and solute migration are decoupled, which implies that inequality in (30) holds for each term separately. Henceforth, we assume that $\bar{\gamma}_{\mathrm{a}}, \pi_{\mathrm{a}}$, and $\mathbf{h}$ are given by

$$
\bar{\gamma}_{\mathrm{a}}(\varsigma, \dot{e})=\frac{\partial \psi(\varsigma)}{\partial e}+a(\varsigma)+b(\varsigma, \dot{e}) \dot{e}, \quad \pi_{\mathrm{a}}(\varsigma, \dot{\varphi})=\mathfrak{a}(\varsigma)+\mathfrak{b}(\varsigma, \dot{\varphi}) \dot{\varphi}
$$

and

$$
\mathbf{h}(\varsigma, \nabla \mu)=-\mathbf{M}(\varsigma, \nabla \mu) \nabla \mu
$$

where, to ensure satisfaction of (30), the response functions $a, b$, $\mathfrak{a}$ and $\mathfrak{b}$ are chosen to be non-negative scalar-valued functions, whereas the mobility response $\mathbf{M}$ is chosen to be a positive-semidefinite tensor function.

Notice that the conditions $b \equiv 0$ and $\mathfrak{b} \equiv 0$ in (31) yield rate-independent responses for $\bar{\gamma}_{\mathrm{a}}$ and $\pi_{\mathrm{a}}$, respectively.

\subsubsection{Yield criterion and the plastic orientation tensor}

The microforce balance (14) and the constitutive theory discussed so far will serve as guides for the introduction of two important pieces of information that are missing: the yield condition, that is, the condition under which plasticity occurs, and the plastic orientation tensor $\mathbf{N}$. This issue has been addressed in Duda et al. (2015) within the context of the classical $J_{2}$-flow theory of plasticity.

We assume that $\gamma^{\text {ext }}=0$ and use the relations $(23)_{2},(24)_{1},(25)$ and $(31)_{1}$ to write the microforce balance (14) as

$$
\tau-y= \begin{cases}\eta \dot{e}, & \dot{e}>0, \\ \overline{\gamma_{\mathrm{r}}}, & \dot{e}=0 .\end{cases}
$$

To simplify the notation, we have introduced the quantities

$$
\eta=b(\varsigma, \dot{e}), \quad y=y_{\mathrm{e}}+y_{\mathrm{d}}, \quad y_{\mathrm{e}}=\frac{\partial \psi(\varsigma)}{\partial e}-\operatorname{Div}\left(\frac{\partial \psi(\varsigma)}{\partial \nabla e}\right), \quad y_{\mathrm{d}}=a(\varsigma)
$$

It is noteworthy emphasizing the distinction between the two alternatives in (33): the first alternative is a restriction on the manner microstructural changes leading to plasticity occur; the second alternative is an identity that determines $\bar{\gamma}_{\mathrm{r}}$ when such changes are arrested -in which case, the microforce balance is automatically satisfied.

It follows from (33) that:

- Plastic flow is driven by the resolved stress $\tau$ and opposed by the plastic-flow resistance $y$, which in turn, has two terms, one energetic $\left(y_{\mathrm{e}}\right)$ and other dissipative $\left(y_{\mathrm{d}}\right)$;

- The conditions $\tau>y$ and $\tau=y$ are necessary for the occurrence of plastic flow when $\eta>0$ and $\eta=0$, respectively. These conditions on the viscosity parameter $\eta$ represent rate-dependent and rate-independent plastic responses;

- The plastic dissipation density, which is defined $\delta_{\mathrm{p}}$ by the first term of (30), can be written as

$$
\delta_{\mathrm{p}}=\left(\tau-y_{\mathrm{e}}\right) \dot{e}
$$

We now turn to the specification of the yield criterion. First, we consider that $\eta>0$. In this case, we already know that $\tau>y$ is necessary for $\dot{e}>0$. Here, we stipulate that this condition is not only necessary but also sufficient for $\dot{e}>0$. Thus, the yield condition states that $\dot{e}>0$ if and only if

$$
\tau>y,
$$

or, equivalently, $\dot{e}=0$ if and only if

$$
\tau \leq y
$$

For a rate-independent plastic response, we know that $\dot{e}>0$ implies $\tau=y$. In this case, we stipulate that (37) must be obeyed, but now with the provision that the strict inequality is sufficient but not necessary for $\dot{e}=0$. Under these conditions, it can be shown that $\dot{e}>0$ if and only if

$$
\tau=y \quad \text { and } \quad \dot{\tau}=\dot{y} \text {. }
$$

Notice that in both rate-dependent and rate-independent case, the reactive microforce $\bar{\gamma}_{\mathrm{r}}$ obeys the inequality

$$
\bar{\gamma}_{\mathrm{r}} \leq 0
$$

Notice that the rate-independent case can be obtained from the rate-dependent one in the limit as $\eta$ goes to zero (c.f. Simo and Hughes, 1998).

Fig. 1 sketches the behavior of $\tau, y, \bar{\gamma}_{\mathrm{r}}$ for a rate independent case in a process where $\dot{e}=0$ and $\dot{e}>0$, at different times, respectively.

To prescribe the plastic orientation tensor $\mathbf{N}$, we substitute $\tau=\mathbf{S}_{0} \cdot \mathbf{N}$ in (35) to write the plastic dissipation as 


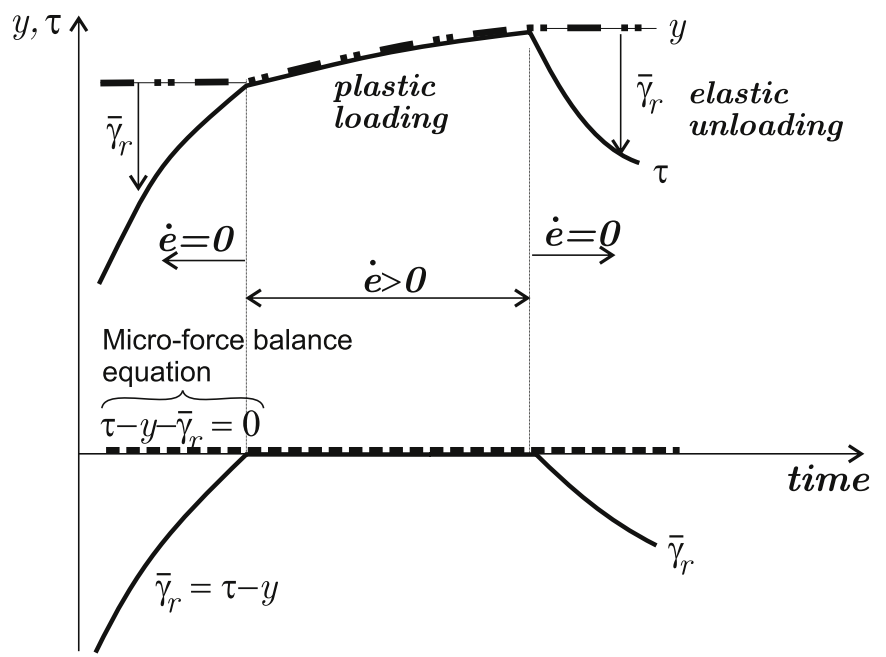

Fig. 1. Resolved stress $\tau$, plastic flow resistance $y$ and reactive plastic micro force $\bar{\gamma}_{\mathrm{r}}$ for a rate independent case.

$$
\delta_{\mathrm{p}}=\left(\mathbf{S}_{0} \cdot \mathbf{N}-y_{\mathrm{e}}\right) \dot{e} \geq 0 .
$$

Notice that (29) $)_{1}$ and $(29)_{2}$ imply that $\mathbf{S}_{0}$, the deviatoric part of $\mathbf{S}$, and $y_{\mathrm{e}}$ are functions of $\boldsymbol{\varsigma}$ and $(\boldsymbol{\varsigma}, \nabla \boldsymbol{\varsigma})$, respectively. Therefore, for given $\varsigma$ and $\nabla \varsigma$, the plastic dissipation is a function of $\mathbf{N}$, whose maximum is attained at

$$
\mathbf{N}=\frac{\mathbf{S}_{0}}{\left|\mathbf{S}_{0}\right|}
$$

and hence

$$
\tau=\left|\mathbf{S}_{0}\right| .
$$

Thus, if the principle of maximum dissipation is invoked, the equations above must hold. See Gurtin et al. (2010) for a detailed discussion of this issue. See also Aifantis (1987).

We finalize this section by collecting the governing equations for $\dot{e}$ and $\bar{\gamma}_{\mathrm{r}}$. For the sake of convenience, let us introduce the function $f$ defined by

$$
f:=\left|\mathbf{S}_{0}\right|-y,
$$

with $y$ given by $(34)_{2}$. In the rate-independent case, it is easy to see that the governing equations for $\dot{e}$ and $\overline{\gamma_{\mathrm{r}}}$ are given by

$$
\dot{e} \geq 0, \quad f \leq 0, \quad \dot{e} f=0, \quad \bar{\gamma}_{\mathrm{r}}=f,
$$

whereas in the rate-dependent case they are given by

$$
\eta \dot{e}=\langle f\rangle, \quad \overline{\gamma_{\mathrm{r}}}=-\langle-f\rangle,
$$

with $\eta$ given by (34) 1 . Henceforth $\langle f\rangle$ denotes the Macaulay bracket of the scalar-valued quantity $f$, that is,

$$
\langle f\rangle= \begin{cases}0, & f \leq 0, \\ f, & f>0 .\end{cases}
$$

\subsubsection{Damage criterion}

Now, we turn to the issue of the damage criterion. The damage criterion adopted here is motivated by the microforce balance (13). The development follows along the same lines adopted for plasticity.

After assuming that $\pi^{\mathrm{ext}}=0$ and using the relations $(24)_{1},(26)$ and $(31)_{2}$, it follows from the microforce balance (13) that

$$
\zeta-\mathfrak{a}= \begin{cases}\mathfrak{b} \dot{\varphi}, & \dot{\varphi}>0, \\ \pi_{\mathrm{r}}, & \dot{\varphi}=0,\end{cases}
$$

where, to simplify the notation, we have introduced the quantity

$$
\zeta:=\operatorname{Div}\left(\frac{\partial \psi(\varsigma)}{\partial \nabla \varphi}\right)-\frac{\partial \psi(\varsigma)}{\partial \varphi} .
$$

Notice that $-\zeta$ is the variational derivative of $\psi$ with respect to $\varphi$. Again, it is important to keep in mind the distinction between the two alternatives in (47): the first alternative is a restriction on the manner microstructural changes leading to damage occur; the 
second alternative is an identity that determines $\pi_{\mathrm{r}}$ when such changes are arrested -in which case, the microforce balance is automatically satisfied. When $\mathfrak{b}>0$, that is, for a rate-dependent damage response, $(47)_{1}$ implies that

$$
\zeta>\mathfrak{a}
$$

is a necessary condition for $\dot{\varphi}>0$. Here, we stipulate that condition is also sufficient for $\dot{\varphi}>0$, which means that damage occurs if and only if (49) holds. Equivalently, no damage occurs whenever

$$
\zeta \leq \mathfrak{a}
$$

is satisfied. For a rate-independent damage response, i.e., for $\mathfrak{b}=0,(47)_{1}$ indicates that $\zeta=\mathfrak{a}$ is a necessary condition for $\dot{\varphi}>0$. In this case, we stipulate that (50) always holds, but now with the provision that the strict inequality is sufficient but not necessary for $\dot{\varphi}=0$. Under these conditions, it can be shown that $\dot{\varphi}>0$ if and only if

$$
\zeta=\mathfrak{a} \text { and } \dot{\zeta}=\dot{\mathfrak{a}} \text {. }
$$

It is easy to see that in both rate-dependent and rate-independent cases, the reactive microforce $\pi_{\mathrm{r}}$ obeys the inequality

$$
\pi_{\mathrm{r}} \leq 0 \text {. }
$$

After introducing the function

$$
F=\zeta-\mathfrak{a},
$$

with $\zeta$ given by (48), it can be shown that the governing equations for $\dot{\varphi}$ and $\pi_{\mathrm{r}}$ reduce to

$$
\dot{\varphi} \geq 0, \quad F \leq 0, \quad \dot{\varphi} F=0, \quad \pi_{\mathrm{r}}=F
$$

in the rate-independent case, and to:

$$
\mathfrak{b} \dot{\varphi}=\langle F\rangle, \quad \pi_{\mathrm{r}}=-\langle-F\rangle
$$

whenever $\mathfrak{b}>0$.

\subsubsection{Structure of the free energy function}

From the constitutive viewpoint, the theory just presented has to be specified by the functions $\psi(\varsigma), \mathbf{M}(\varsigma, \nabla \mu), a(\varsigma), b(\varsigma, \dot{e}), \mathfrak{a}(\varsigma)$, and $\mathfrak{b}(\varsigma, \dot{\varphi})$, with $\varsigma=\left(\mathbf{E}_{\mathrm{e}}, e, \varphi, c, \nabla e, \nabla \varphi\right)$. Through these functions, various solid-solute interactions can be incorporated in the theory. In particular, mutual solid-solute interactions can be implemented via the free energy response.

Therefore, we consider the free energy response given by

$$
\psi\left(\mathbf{E}_{\mathrm{e}}, e, \varphi, c, \nabla e, \nabla \varphi\right)=\psi_{e}\left(\mathbf{E}_{\mathrm{e}}, c, \varphi\right)+\psi_{p}(e, \nabla e, c, \varphi)+\psi_{f}(\varphi, \nabla \varphi, c)+\psi_{c}(c),
$$

where $\psi_{e}, \psi_{p}, \psi_{f}$ and $\psi_{c}$ stand for elastic, plastic, fracture and chemical free energies. The first three contributions, which represent the energy stored in the host solid, appear as a result of the stretching of atomic bonds $\left(\psi_{e}\right)$ and the presence of defects generated during plastic flow $\left(\psi_{p}\right)$ and bond breakage $\left(\psi_{f}\right)$. As (56) shows, we allow that these contributions can be affected by the presence of the guest solute and that the elastic and plastic energies can depend on the fraction of broken bonds $\varphi$. In particular, it is natural to assume that $\psi_{e}$ is a decreasing function of $\varphi$, that is, $\partial \psi_{e} / \partial \varphi<0$. The chemical contribution $\psi_{c}$ represents the free energy of the interstitial solute species in the host solid.

After combining $(23)_{1},(29)_{4}$ and (56), we arrive at the following expression for the chemical potential $\mu$ :

$$
\mu=\mu_{c}+\mu_{e}+\mu_{p}+\mu_{f}+\mu_{\sigma}
$$

where

$$
\mu_{c}=\frac{\partial \psi_{c}}{\partial c}, \quad \mu_{e}=\frac{\partial \psi_{e}}{\partial c}, \quad \mu_{p}=\frac{\partial \psi_{p}}{\partial c}, \quad \mu_{f}=\frac{\partial \psi_{f}}{\partial c}, \quad \mu_{\sigma}=-v \sigma_{\mathrm{m}}
$$

Since solute migration is driven by the gradient of the chemical potential, each one of the terms above provides a driving force for solute migration. In particular, the gradients of $\mu_{c}$ and $\mu_{\sigma}$ give rise to the driving forces provided by the concentration and mean-stress gradients. Furthermore, spatial variations of the fields $\mathbf{E}_{\mathrm{e}}, e$ and $\varphi$, can provide new driving forces for solute migration. Therefore, whenever the guest solute affects the host solid by inducing deformation and changes in the elastic, plastic and fracture properties appearing in the corresponding free energies, the host solid affects the guest solute by promoting migration in response to inhomogeneity in its state of stress, elastic strain, plastic strain and fracture.

The solute effects described above give rise to a two-way coupling between solute migration and elastic-plastic deformation and fracture behavior of the host solid. However, the solute can also affect the elastic-plastic and fracture behavior of the host solid via the dissipative responses $a, b, \mathfrak{a}$ and $\mathfrak{b}$, which give rise to one-way couplings between solute migration and elastic-plastic deformation and fracture of the host solid. On the other hand, the host solid can influence solute migration via the mobility response $\mathbf{M}$, which again is a one-way coupling. From now on, we assume that the mobility response of the solute flux is given by

$$
\mathbf{h}=-M c \nabla \mu
$$


where $M(\varsigma)>0$ is the solute mobility in the host solid.

Notice that $M$ can depend on the stress state $\mathbf{S}$, see the discussion of Larché and Voorhees (1996). The same applies to $a$.

\subsection{Governing equations}

Now, we collect the results obtained so far to write the set of governing equations for the basic fields of the theory, namely $\mathbf{u}, c, e$ and $\varphi$. This set of equations is obtained by combining the force balances and constitutive theory, including the yield and damage criteria.

- Displacement field $\mathbf{u}$ :

$$
\begin{aligned}
& \operatorname{Div} \mathbf{S}+\mathbf{b}=0, \quad \mathbf{S}=\frac{\partial \psi_{e}}{\partial \mathbf{E}_{\mathrm{e}}}, \\
& \mathbf{E}=\mathbf{E}_{\mathrm{e}}+\mathbf{E}_{\mathrm{p}}+\mathbf{E}_{\mathrm{c}}, \\
& \dot{\mathbf{E}}_{\mathrm{p}}=\dot{e} \frac{\mathbf{S}_{0}}{\left|\mathbf{S}_{0}\right|}, \quad \mathbf{E}_{c}=\frac{v}{3}\left(c-c_{0}\right) \mathbf{I} .
\end{aligned}
$$

- Solute content $c$ :

$$
\begin{gathered}
\dot{c}=-\operatorname{Divh}, \quad \mathbf{h}=-M c \nabla \mu, \\
\mu=\frac{\partial\left(\psi_{c}+\psi_{e}+\psi_{p}+\psi_{f}\right)}{\partial c}-v \sigma_{\mathrm{m}} .
\end{gathered}
$$

- Accumulated plastic strain $e$ :

$$
\begin{aligned}
& b \dot{e}=\langle f\rangle \quad \text { (rate-dependent response) } \\
& \dot{e} \geq 0, \quad f \leq 0, \quad \dot{e} f=0 \quad \text { (rate-independent response) } \\
& f=\left|\mathbf{S}_{0}\right|-\left(\frac{\partial \psi_{p}}{\partial e}+a\right)+\operatorname{Div} \tau, \quad \tau=\frac{\partial \psi_{p}}{\partial \nabla e} .
\end{aligned}
$$

- Phase field $\varphi$ :

$$
\begin{aligned}
& \mathfrak{b} \dot{\varphi}=\langle F\rangle \quad(\text { rate }- \text { dependent response }) \\
& \dot{\varphi} \geq 0, \quad F \leq 0, \quad \dot{\varphi} F=0 \quad(\text { rate }- \text { independent response) } \\
& F=\operatorname{Div} \xi-\left(\frac{\partial\left(\psi_{e}+\psi_{p}+\psi_{f}\right)}{\partial \varphi}+\mathfrak{a}\right), \quad \xi=\frac{\partial \psi_{f}}{\partial \nabla \varphi}
\end{aligned}
$$

At this point it is expedient to discuss the following couplings that can be incorporated in the theory:

\section{- Elasticity and fracture}

The interaction of elastic deformation and fracture can be implemented by allowing an elastic free energy $\psi_{e}$ that decreases with $\varphi$. In this case, $\partial \psi_{e} / \partial \varphi<0$ provides a driving force for bond breakage, as (63) $)_{3}$ shows. In this way, several driving forces can be incorporated depending on how $\psi_{e}$ depends on $\varphi$. Additional interactions can be incorporated by allowing constitutive dependency of the viscous modulus $\mathfrak{b}$ and dissipative resistance $\mathfrak{a}$ on the elastic strain $\mathbf{E}_{\mathrm{e}}$. Therefore, the elastic strain can affect not only the damage driving force but also the damage resistance. This allows, for instance, to consider that damage is frozen under compressive stresses by selecting $\mathfrak{a}$ as a function of $\mathbf{E}_{\mathrm{e}}$ in an appropriate manner.

- Plasticity and fracture

The interaction of plastic deformation and fracture can be implemented by allowing a damage-dependent plastic free energy $\psi_{p}$. Notice that if $\psi_{p}$ decreases with $\varphi$, for example the plastic hardening decreasing with $\varphi$, then $\partial \psi_{p} / \partial \varphi<0$ provides an additional driving force for bond breakage, as $(63)_{3}$ shows. Additional interactions can be incorporated by allowing constitutive dependency of the viscous modulus $b$ and dissipative resistance $a$ on the elastic strain $\mathbf{E}_{\mathrm{e}}$.

- Elasticity and solute migration

The interaction of elastic deformation and solute migration can be implemented by allowing a composition-dependent elastic free energy $\psi_{e}$. In this case, the gradient of $\partial \psi_{e} / \partial c$ provides a driving force for solute migration, as (61) shows. Additional interactions can be incorporated by allowing constitutive dependency of the mobility $M$ on the elastic strain $\mathbf{E}_{\mathrm{e}}$. 
- Plasticity and solute migration

The interaction of plastic deformation and solute migration can be implemented by allowing a composition-dependent plastic free energy $\psi_{p}$. In this case, the gradient of $\partial \psi_{p} / \partial c$ provides a driving force for solute migration, as (61) shows. Additional interactions can be incorporated by allowing constitutive dependency of the mobility $M$ on $e$ and $\nabla e$ and of the viscous modulus $b$ and dissipative resistance $a$ on $c$.

- Fracture and solute migration

The interaction of fracture and solute migration can be implemented by allowing a composition-dependent fracture free energy $\psi_{f}$ . In this case, the gradient of $\partial \psi_{f} / \partial c$ provides a driving force for solute migration, as (61) shows. Additional interactions can be incorporated by allowing constitutive dependency of the mobility $M$ on $\varphi$ and $\nabla \varphi$ and of the viscous modulus $\mathfrak{b}$ and dissipative resistance $\mathfrak{a}$ on $c$.

\section{Specialization of the model equations for the hydrogen-assisted cracking problem}

Hydrogen-assisted cracking (HAC) is a highly complex phenomenon by which host metallic solids under stress undergo premature fracture due to the presence of guest hydrogen atoms. Numerous mechanisms have been invoked for its explanation, including the one known as hydrogen-enhanced decohesion which presumes that the presence of guest hydrogen atoms in the host solid reduces its resistance to bond breakage.

To account for this specific aspect of HAC, we consider a simple particularization of the general theory presented before based on the following constitutive assumptions:

- Local plasticity is assumed.

- The elastic free energy density $\psi_{e}$ is given by

$$
\psi_{e}\left(\mathbf{E}_{\mathrm{e}}, \varphi\right)=(1-\varphi)^{2} \psi_{e}^{0}\left(\mathbf{E}_{\mathrm{e}}\right)
$$

where

$$
\psi_{e}^{0}\left(\mathbf{E}_{\mathrm{e}}\right)=\frac{\lambda}{2}\left(\operatorname{tr} \mathbf{E}_{\mathrm{e}}\right)^{2}+G\left|\mathbf{E}_{\mathrm{e}}\right|^{2}
$$

is the elastic free energy density of a pristine host solid, with $\lambda$ and $G$ the corresponding Lamè moduli;

- The chemical free-energy density $\psi_{c}$ is given by

$$
\psi_{c}(c)=\mu_{0} c+R T c(\ln c-1),
$$

which involves the free energy of mixing of hydrogen atoms diluted in the interstitial sites of the host lattice ${ }^{1}$, where $R$ is the universal gas constant and $T$ the absolute temperature;

- The plastic free-energy density $\psi_{p}$ and the dissipative resistance $a$ are given by

$$
\psi_{p}(e)=\frac{\sigma_{y}^{2}}{E(n+1)}\left(\left(1+\sqrt{\frac{2}{3}} \frac{e}{\varepsilon_{0}}\right)^{n+1}-1\right)-\sqrt{\frac{2}{3}} \sigma_{y} e, \quad a=\sqrt{\frac{2}{3}} \sigma_{y}
$$

where $E$ is the Young modulus, $\sigma_{y}$ the yield stress, $n$ the hardening parameter, $\varepsilon_{0}=\sigma_{y} / E$ the uniaxial strain at the yield stress. The viscous modulus $b$ vanishes;

- The fracture free-energy density $\psi_{f}$ is given by

$$
\psi_{f}(\varphi, \nabla \varphi, c)=g_{f}(c)\left(\frac{\varphi^{2}}{2 l}+\frac{l}{2}|\nabla \varphi|^{2}\right),
$$

where $g_{f}(c)$, a decreasing function of $c$, and $l$ are the fracture energy and the characteristic length of the phase field model. The dissipative resistance $\mathfrak{a}$ and viscous modulus $\mathfrak{b}$ are neglected.

We would like to stress that our purpose here is to come up with a simple theory capable of accounting for the negative effect of

\footnotetext{
${ }^{1}$ This expression can be obtained as follows. For an ideal solution of hydrogen atoms dissolved in the lattice sites of a host metal, the chemical free energy density can be written $\operatorname{as} \psi_{c}(c)=\mu_{0}^{*} c+R T N(\theta \ln \theta+(1-\theta) \ln (1-\theta))$ where $\mu_{0}^{*}$ is a reference chemical potential, $N$ the number of moles of lattice sites per unit of solution volume, and $\theta=c / N$ the occupancy fraction of lattice sites (e.g. Di Leo and Anand, 2013). When $\theta \ll 1(c \ll N)$, which corresponds to a dilute solution, the chemical free energy admits the approximation $\psi_{c}(c) \approx \mu_{0}^{*} c+R T c(\ln (c / N)-1)=\left(\mu_{0}^{*}-R T \ln N\right) c+R T c(\ln c-1)$, which furnishes the result $(66)$ after defining $\mu_{0}:=\mu_{0}^{*}-R T \ln N$.
} 
guest hydrogen atoms on the resistance to bond breakage of the host solid. In particular, we assume that the plastic response is affected neither by the phase field nor by the hydrogen content.

With the aforementioned constitutive choices, the set of governing equations for $\mathbf{u}, c, e$ and $\varphi$ simplifies to:

- Displacement field $\mathbf{u}$ :

$$
\begin{aligned}
& \operatorname{Div} \mathbf{S}+\mathbf{b}=0, \quad \mathbf{S}=\left((1-\varphi)^{2}\right)\left(\lambda\left(\operatorname{tr} \mathbf{E}_{e}\right) \mathbf{I}+2 G \mathbf{E}_{e}\right), \\
& \mathbf{E}=\mathbf{E}_{\mathrm{e}}+\mathbf{E}_{\mathrm{p}}+\mathbf{E}_{\mathrm{c}}, \\
& \dot{E}_{\mathrm{p}}=\dot{e} \frac{\mathbf{S}_{0}}{\left|\mathbf{S}_{0}\right|}, \quad \mathbf{E}_{c}=\frac{v}{3}\left(c-c_{0}\right) \mathbf{I} ;
\end{aligned}
$$

- Atomic hydrogen molar density $c$ :

$$
\begin{aligned}
& \dot{c}=-\operatorname{Divh}, \quad \mathbf{h}=-M c \nabla \mu, \\
& \mu=\underbrace{\mu_{0}+R T \ln c}_{\mu_{c}}+\underbrace{g_{f}^{\prime}(c)\left(\frac{\varphi^{2}}{2 l}+\frac{l}{2}|\nabla \varphi|^{2}\right)}_{\mu_{f}}-\underbrace{v \sigma_{\mathrm{m}}}_{-\mu_{\sigma}} ;
\end{aligned}
$$

- Accumulated plastic strain $e$ :

$$
\begin{aligned}
& \dot{e} \geq 0, \quad f \leq 0, \quad \dot{e} f=0, \\
& f=\left|\mathbf{S}_{0}\right|-\sqrt{\frac{2}{3}} \sigma_{y}\left(1+\sqrt{\frac{2}{3}} \frac{e}{\varepsilon_{0}}\right)^{n} ;
\end{aligned}
$$

- Phase field $\varphi$ :

$$
\begin{aligned}
& \dot{\varphi} \geq 0, \quad F \leq 0, \quad \dot{\varphi} F=0, \\
& F=2(1-\varphi) \psi_{e}^{0}\left(\mathbf{E}_{\mathrm{e}}\right)-\frac{1}{l}\left(g_{f}(c) \varphi-l^{2} \operatorname{Div}\left(g_{f}(c) \nabla \varphi\right)\right) .
\end{aligned}
$$

The following remarks follow from the constitutive choices above:

- Plastic flow is driven by $\mathbf{S}_{0}$ and opposed by a term that depends on the accumulated plastic strain $e$. In particular, the resistance to plastic flow does not depend of the amount of broken bonds and hydrogen atoms. Further, during plastic flow the following relation holds:

$$
\left|\mathbf{S}_{0}\right|=\sqrt{\frac{2}{3}} \sigma_{y}\left(1+\sqrt{\frac{2}{3}} \frac{e}{\varepsilon_{0}}\right)^{n} ;
$$

- Bond breakage is driven by $2(1-\varphi) \psi_{e}^{0}\left(\mathbf{E}_{\mathrm{e}}\right)$ or, equivalently, by

$$
\frac{2}{(1-\varphi)^{3}}\left(\frac{\sigma_{\mathrm{m}}^{2}}{2 K}+\frac{\left|\mathbf{S}_{0}\right|^{2}}{G}\right),
$$

where $K=(3 \lambda+2 G) / 3$ is the bulk modulus of the pristine material, and opposed by the resistance

$$
\frac{g_{f}(c)}{l}\left(\varphi-l^{2} \triangle \varphi\right)+l^{2}\left|g_{f}^{\prime}(c)\right|(\nabla \varphi \cdot \nabla c)
$$

where the condition $g_{f}^{\prime}(c)<0$ has been used;

- Since $\mathbf{h}=-M c \nabla \mu$ and $M>0$, hydrogen atoms tend to move from a region of high chemical potential to a region of low chemical potential. Thus, in view of the fact that $\mu=\mu_{c}+\mu_{f}+\mu_{\sigma}$ (since $\mu_{e}=\mu_{p}=0$ ), hydrogen atoms tend to move from a region of high concentration to a region of low concentration and from a region of low mean stress (resp. fracture energy) to a region of high mean stress (resp. fracture energy).

\section{Model assessment}

We now consider a numerical application of the theory introduced in the previous Section. Towards this end, we propose a numerical model based on a variational formulation of the theory and its implementation using the finite-element method for spatial discretization and a splitting scheme with sub-stepping for the time integration. Details concerning the development of the numerical model are provided in Appendix I.

Our purpose in this Section is to evaluate the capability of the theory, and the corresponding numerical implementation, to capture, at least qualitatively, aspects of the hydrogen-assisted cracking behavior of steels when exposed to an aggressive 


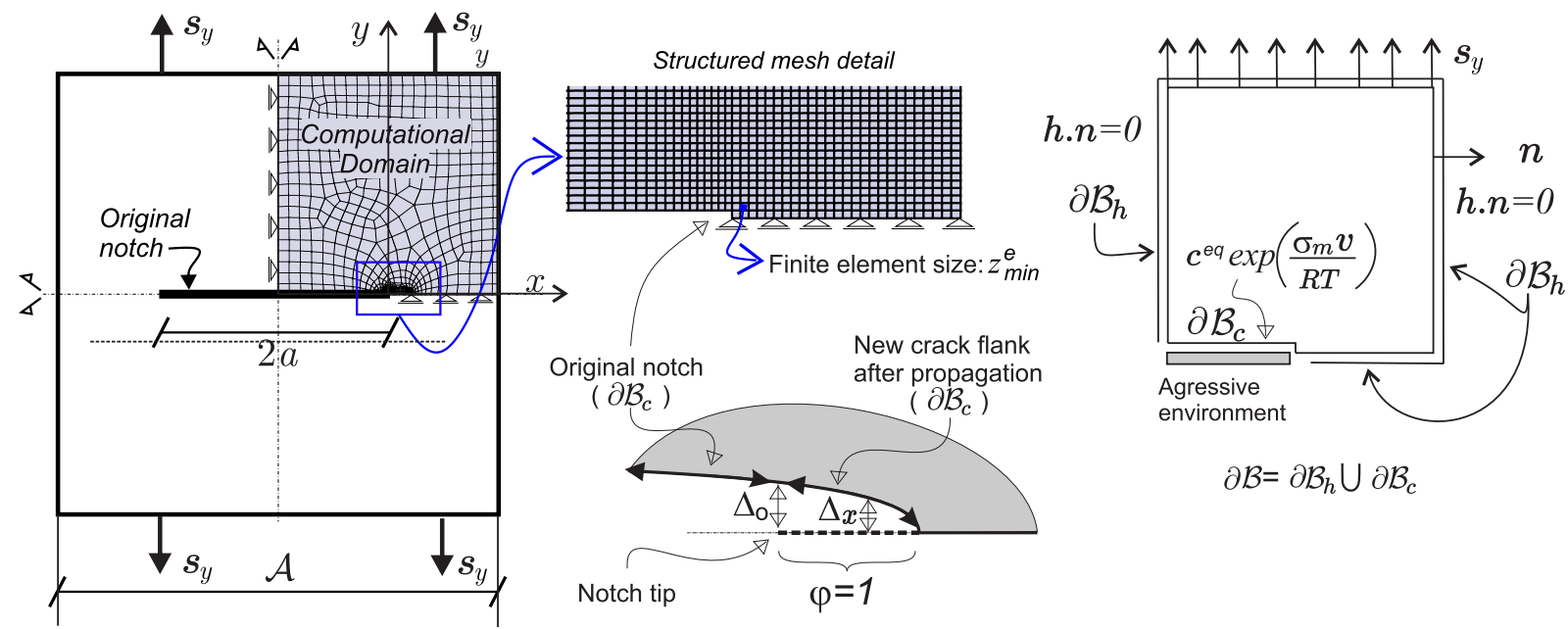

Fig. 2. Center sharp-notched panel test. Geometrical model and boundary conditions for displacements and diffusion of hydrogen.

environment supplier of hydrogen atoms. This problem has been considered by a number of authors. In particular, Serebrinsky et al. (2004) has presented a set of numerical simulations using an interface cohesive (traction-separation) model, coupled with plasticity and hydrogen diffusion. In the approach of Serebrinsky et al., the failure mechanism is assumed to be the degradation of the cohesive properties of steel caused by the presence of interstitial hydrogen atoms. We refer the reader to Jemblie et al. (2017) for a comprehensive review on the use of cohesive zone modeling to address hydrogen-assisted cracking in steels.

\subsection{Hydrogen embrittlement test}

We simulate the crack initiation and propagation problem in several center-notched panels under sustained loads depicted in Fig. 2-a. The panels are in contact with an aggressive environment working as a source of hydrogen atoms.

The three most salient effects simulated here are:

(i) Hydrogen uptake from the environment through the panel notch and crack flanks by defining appropriate boundary conditions for the hydrogen concentration variable;

(ii) stress-assisted hydrogen diffusion; and

(iii) hydrogen embrittlement characterized by means of hydrogen-induced reduction of the fracture energy.

\subsubsection{Problem description}

The panel geometry and load conditions are shown in Fig. 2. Relevant dimensions are $\mathscr{A}=20 \mathrm{~mm}$ and $2 a=10 \mathrm{~mm}$. Plane strain conditions are assumed. The panel is loaded by a uniform and constant traction $s_{y}$. In the following, the load level $s_{y}$ is identified with the corresponding stress intensity factor $K_{I}$ computed analytically at the original notch tip, see Anderson (2005). The hydrogen source comes from considering an aggressive aqueous environment existing in the interior of the notch.

Three high-strength steels, henceforth denoted by Steels A, B and C, are considered in the simulations. They are characterized by the same elastic properties (Young modulus $E=210 \mathrm{GPa}$, Poisson's ratio $\nu=0.3$ ) and different parameters $\sigma_{y}, n, K_{I c}^{o}$, and $l$, which are indicated in Table 1. The fracture energy of the hydrogen-free steel $g_{f}^{o}=g_{f}(0)$ is computed from the corresponding fracture toughness $K_{I c}^{o}$ through the relation: $g_{f}^{o}=\left(1-v^{2}\right)\left(K_{I c}^{o}\right)^{2} / E$.

Excluding the phase-field characteristic length parameter $l$, all the remaining parameters in Table 1 are taken from Serebrinsky et al. (2004), which is taken as a reference work for comparing our results. The parameter $l$ is estimated as discussed below.

Keeping in mind that we want to obtain comparable results with those presented by Serebrinsky et al., we recall that one of the most important parameters governing the cohesive fracture model in Serebrinsky et al. is the peak stress $\sigma_{c}$ of the cohesive law.

Table 1

Center sharp-notched panel test. Mechanical properties of three steels. $\sigma_{y}$ : yield stress, $n$ : hardening exponent of the plastic law (eq. (71)), $K_{I c}^{o}$ : fracture toughness, $l$ : characteristic length of the phase field model $\left(\sigma_{y}, n\right.$ and $K_{I c}^{o}$ taken from Serebrinsky et al., 2004.

\begin{tabular}{llll}
\hline & Steel A & Steel B & Steel C \\
\hline$\sigma_{y}[\mathrm{MPa}]$ & 1200 & 1400 & 1600 \\
$n$ & 0.064 & 0.052 & 0.043 \\
$K_{I c}^{o}[\mathrm{MPa} \sqrt{m}]$ & 100 & 75 & 52 \\
$l[\mu \mathrm{m}]$ & $150-200$ & 50 & 50
\end{tabular}


Table 2

Center sharp-notched panel test. Hydrogen diffusion properties. $D_{\text {eff }}$ : effective diffusion coefficient, $v$ : partial molar volume of hydrogen in Fe, $\triangle g_{b}$ : energy parameter used in equation (76), $R$ : universal gas constant and T: ambient temperature (data taken from Serebrinsky et al., 2004).

\begin{tabular}{llll}
\hline$M R T=D_{\text {eff }}\left[\mathrm{m}^{2} / \mathrm{s}\right]$ & $v\left[\mathrm{~m}^{3} / \mathrm{mol}\right]$ & $\Delta g_{b}[\mathrm{~kJ} / \mathrm{mol}]$ & $R\left[\mathrm{~J} / \mathrm{mol} \mathrm{o}^{\circ} \mathrm{K}\right]$ \\
\hline $10^{-10}$ & $2 . \times 10^{-6}$ & 30 & 29314 \\
\hline
\end{tabular}

According to Tvergaard and Hutchinson (1992), the peak stress of cohesive models may be related to the yield stress by $\sigma_{c}=4 \sigma_{y}$. This factor 4 is an approximated value defining the peak stress of cohesive interface elements for capturing the coupled effects of plasticity and crack propagation. Alternatively, in phase field models, there is not a material parameter characterizing the peak stress, $\sigma_{c}^{P F}$, of the damage law. However, as shown by Duda et al. (2015), it can be estimated with the formula

$$
\sigma_{c}^{P F}=\frac{3 \sqrt{3}}{16} \sqrt{\frac{g_{f}^{o} E}{l}}=\frac{3 \sqrt{3}}{16} \sqrt{\frac{1-v^{2}}{l}} K_{I c}^{o} .
$$

Then, the characteristic lengths $l$ shown in Table 1 have been determined using this formula and adopting a correspondence $\sigma_{c}^{P F}=\kappa \sigma_{y}$, where $\kappa$ is in the range $1.4 \leq \kappa \leq 2.4$.

Table 2 describes the parameters related to the hydrogen diffusion equation.

\subsubsection{Initial and boundary conditions.}

- The initial condition for damage is $\varphi(\mathbf{x}, 0)=0$ for all $\mathbf{x}$ in $\mathcal{B}$, which means that the complete panel is initially pristine. As for the boundary condition, we consider that the normal gradient is prescribed to zero on the full boundary, $\mathbf{n} \cdot \nabla \varphi(\partial \mathcal{B}, t)=0$, including the crack flanks during the crack propagation stage, see inset in Fig. 2.

- The initial condition for the bulk hydrogen concentration is $c(\mathbf{x}, 0)=c_{0}=0$ for all $\mathbf{x}$ in $\mathcal{B}$, which means that the complete panel is initially hydrogen-free. On the boundary $\partial \mathcal{B}_{h}$, excluding the notch and crack flanks, the hydrogen flux is fixed to zero: $\mathbf{n} \cdot \boldsymbol{h}\left(\partial \mathcal{B}_{h}, t\right)=0$. The notch and crack flank surfaces, $\partial \mathcal{B}_{c}$, are assumed to be in contact with an aggressive environment whose hydrogen chemical potential is $\mu_{\text {env }}$. Considering that the chemical potential on the metal boundary is in equilibrium with the environment, $\mu\left(\partial \mathcal{B}_{c}, t\right)=\mu_{\text {env }}$, and using $(70)_{3}$, the bulk hydrogen concentration on $\partial \mathcal{B}_{c}$, results:

$$
c\left(\partial \mathcal{B}_{c}, t\right)=c^{e q} \exp \left(\frac{\sigma_{m} v}{R T}\right) \underbrace{\exp \left(-\frac{g_{f}^{\prime}(c)}{2 l R T}\left(\varphi^{2}+l^{2}|\nabla \varphi|^{2}\right)\right)}_{\chi_{g_{f}}}
$$

where $c^{e q}=\exp \left(\left(\mu_{\mathrm{env}}-\mu_{0}\right) / R T\right)$ is an specified hydrogen concentration in equilibrium with the aggressive environment. Following Serebrinsky et al. (2004), we take $c^{e q}=5.5 \times 10^{-6}$ (in $\left.\mathrm{mol} / \mathrm{mol}\right)^{2}$, which corresponds to a $3 \% \mathrm{NaCl}$ aqueous solution, and assume that $\chi_{g_{f}}=1$. Note that the boundary $\partial \mathcal{B}_{c}$ changes as the crack propagates. Additional details about the imposition of condition (74) during the crack propagation stage are given in the following sub-Sections.

\subsubsection{Hydrogen embrittlement model}

The effect of hydrogen on the metal cohesion is of preponderant importance for simulating environmental-assisted cracking. The modeling of this complex phenomena requires sophisticated tools of analysis which are beyond the scope of the present contribution.

Here, instead, a macroscopic model to simulate the metal embrittlement due to hydrogen is introduced by following a rather straight description given in the paper of Serebrinskly et al., see Section 2 of that reference work. The steel toughness degradation is defined by means of the fracture energy law

$$
g_{f}(c)=g_{f}^{o} \gamma(c)^{p}
$$

where $g_{f}^{o}$ is the fracture energy of the pristine steel obtained from the specified fracture toughness, as mentioned in Table 1 . The degradation function $\gamma(c)$, with $0 \leq \gamma(c) \leq 1$, depends on the hydrogen concentration as follows

$$
\gamma(c)=1-1.0467 \theta(c)+0.1687 \theta(c)^{2} \quad ; \quad \theta(c)=\frac{c}{c+\exp \left(-\Delta g_{b} / R T\right)}
$$

The coverage fraction $\theta$ relates the hydrogen surface concentration and its saturation value through the Langmuir-McLean isotherm given by the last identity in (76). In this expression, $\Delta g_{b}$ is the free energy difference between the adsorbed and bulk standard states (see Table 2). The exponent $p$ in expression (75) is a parameter to be adjusted according to a procedure explained in the following paragraphs.

The embrittlement law (75) with the exponent $p=1$, jointly with the expressions in (76), has been used by Serebrinsky et al.

\footnotetext{
${ }^{2}$ By taking the partial molar volume of the host lattice, $v=6.87 \times 10^{3} \mathrm{~mm}^{3} / \mathrm{mol}$ (from Lufrano et al., 1998), the equilibrium hydrogen concentration is expressed as $c^{e q}=8 . \times 10^{-10} \mathrm{molH}_{1} / \mathrm{mm}^{3}$.
} 
Based on experimental results, these authors argument in favor of an embrittlement law displaying saturation at high hydrogen concentration. The $\theta$-function in (76) shows this behavior. They also fit the function $\gamma(c)$ from previous experimental results, and they additionally assume that the cohesive model critical stress is linearly degraded with $\gamma(c)$. Further details and discussion about equations (75) and (76) can be found in this reference work.

In the present phase field model, recalling that the critical stress magnitude $\sigma_{c}^{P F}$ is estimated with (73) and assuming that $E$ and $l$ are fixed parameters for a given test, $\sigma_{c}^{P F}$ cannot be modeled with an identical proportional degradation to that defined for the parameters $g_{f}(c)$. So, in order to attain a fracture degradation model comparable to that reported in Serebrinsky et al., we fit the parameter $p$, in the range $1 \leq p \leq 2$, to match a specific result taken from this reference work. This procedure is further explained in following paragraphs.

Recent additional discussion on hydrogen degradation laws in the context of the hydrogen enhanced decohesion mechanism (HEDE) has been reported by Yu et al. (2016) and references cited therein.

\subsubsection{Finite element model}

A structured mesh of B-Bar quadrilateral finite elements is used around the notch tip. Taking advantage of the problem symmetry, only a fourth part of the plate is simulated, such as shown in Fig. 2. The finite element size of the structured mesh close to the notch tip is $z_{\text {min }}^{e}=5 \mu m$.

With this mesh and integration time steps of the order 1 to $10 \mathrm{sec}$, the computed hydrogen concentration fields, close to the notch root, display stable and smooth numerical solutions.

In a previous work, see Sections 4.1.2 and 4.1.3 of Duda et al. (2015), the authors have evaluated the independence of the numerical results provided by the phase-field model with respect to the finite element mesh size. The same conclusions can be directly translated from there to the present situation.

\subsubsection{Analysis of results}

All simulated tests of hydrogen-assisted cracking display two well-differentiated stages.

In the first stage, denoted Stationary stage (St), due to the fact that the loads are lower than the critical ones $\left(K_{I}<K_{I c}^{o}\right)$, the notch tip remains stationary while hydrogen uptake is taking place through the notch flanks and diffuses across the specimen bulk.

In a second stage, denoted Propagating stage (Pr), as hydrogen builds up in the region close to the notch tip, assisted by the high mean stresses, the material toughness decreases. At time $t_{c}$, when the fracture energy has decreased to a low critical value, a crack is formed and propagates instantaneously until reaching the length $\ell_{c}$. At fixed load, this process is unstable. After that, at $t>t_{c}$, the crack propagation process stabilizes and the crack tip propagates at an almost constant rate $v_{c}$.

In accordance with this description, results are next analyzed with particular attention to the three variables $t_{c}, \ell_{c}$ and $v_{c}$, which can be evaluated using the information provided by the numerical results and summarized in Fig. 3.

Fig. 3 represents the distribution of the phase field at a certain time, during the stage Pr. We assume that the point $x_{f}$ ahead of the crack, satisfying the condition $\varphi>0.97$, identifies the crack tip at different times. We also assume that the point $x_{v}$ ahead of the crack tip with a damage value $\varphi=0.05$ identifies the boundary of the damaged zone. Then, the crack tip propagation rate $v_{c}$ is related to the speed of the point $x_{f}$, while $t_{c}$ is the time at which the crack initiates and $\ell_{c}$ the length from $x_{f}$ to the notch root at $t_{c}$. In Fig. 3 , we denote $r_{f}$ and $r_{v}$ the distances from the notch root to the points $x_{f}$ and $x_{v}$, respectively. Two plastic bulbs are also observed in the Figure. They are characterized with the sizes $l_{p}^{0}$ and $l_{p}^{1}$ respectively. The size $l_{p}^{0}$ is the width, along the $\mathrm{x}$-axis, of the initial plastic bulb located around the notch root, while $l_{p}^{1}$ is the distance from the notch root to the point where the second plastic bulb initiates at stage Pr.

The numerical integration of the evolution equations governing plasticity and damage at the time step $t_{c}$, during the unstable crack propagation stage, has to be computed carefully. A load control technique does not work for computing the unstable process triggered at this time step. Instead, the crack length $\ell_{c}$ is evaluated by computing numerous mechanical loops, at fixed time step such

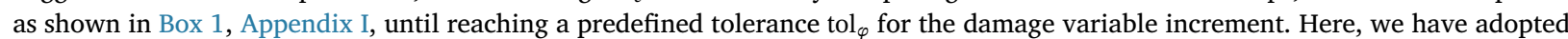
the tolerance value $\operatorname{tol}_{\varphi}=0.002$.

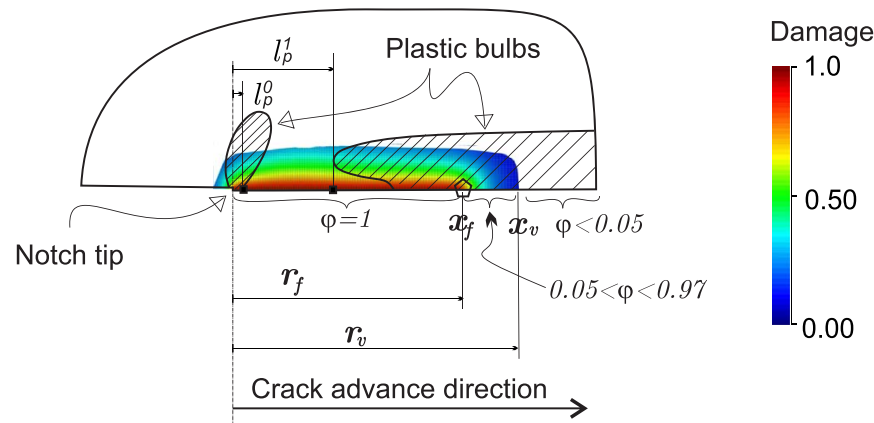

Fig. 3. Center sharp-notched panel test. Identification of the information provided by the numerical model. 


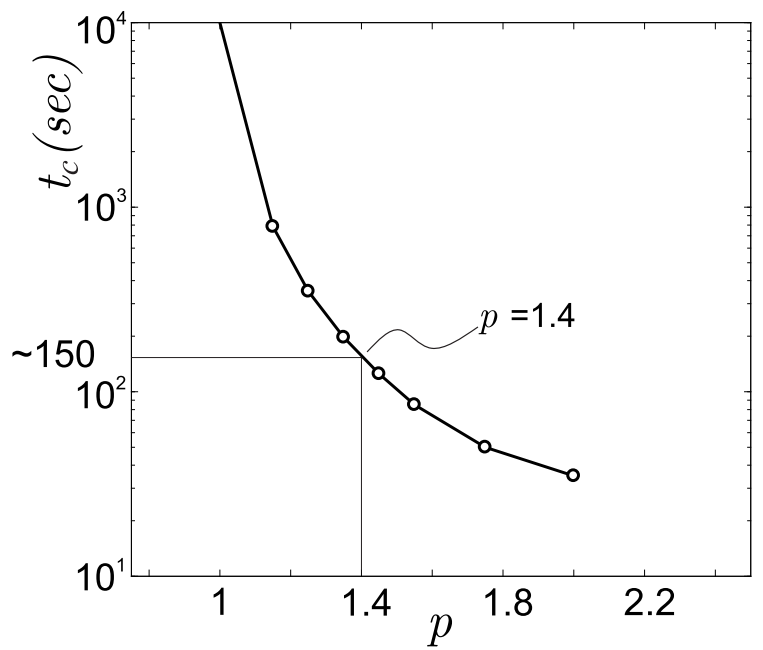

Fig. 4. Time to crack initiation $t_{c}$ vs. degradation function exponent $p$. Simulated test: Steel B, $\sigma_{y}=1400 \mathrm{MPa}, K_{I}=60 \mathrm{MPa} \sqrt{m}$ (reference value $t_{c} \approx 150 \mathrm{sec}$ taken from Serebrinsky et al.).

4.1.4.1. Dependence of the crack initiation time with the degradation function. Fig. 4 plots the crack initiation time, $t_{c}$, vs. the degradation function exponent $p$ defined in expression (77) obtained with the simulation of the Steel $\mathrm{B}, K_{I}=60 \mathrm{MPa} \sqrt{m}$ and $l=50 \mu \mathrm{m}$. For the same Steel and load, Serebrinsky et al. reports a value $t_{c} \approx 150 \mathrm{sec}$. In accordance with Fig. 4, the time to crack initiation computed with $p=1.4$ approximately coincides with the reference value. As our goal is to compare our results with those of Serebrinsky et al., we choose this value of the exponent $p$ for describing the degradation function for all the following simulations, independently of steels and loads.

4.1.4.2. Evaluation of different alternatives for prescribing hydrogen fluxes on the new generated crack flanks. Results simulated with Steel B, load $K_{I}=50 \mathrm{MPa} \sqrt{m}, p=1.4$ and two types of boundary conditions are next analyzed. These two boundary conditions correspond to different ways of instantaneously prescribing the hydrogen concentration, defined by equation (74), on the crack flanks generated during the propagation stage. They are denoted by $\mathrm{Bc} 1$ and $\mathrm{Bc} 2$ and are defined as follows:

Bc1: Boundary condition (74) is prescribed on nodes that are intersected by the crack flanks and satisfies $\Delta_{x}>0.4 \Delta_{0}$, where $\Delta_{x}(t)$ is the crack opening displacement at the corresponding mesh node and $\Delta_{0}(t)$ is the crack opening displacement at the original notch tip, see inset of Fig. 2.

Bc2: Boundary condition (74) is prescribed on nodes that are intersected by the crack flanks and satisfies $\varphi>0.97$.

All nodes intersected by the crack flanks, satisfy the criterion to activate Bc1 after that required for activating the condition Bc2. Therefore, boundary condition $\mathrm{Bc} 1$, if compared with $\mathrm{Bc} 2$, delays the hydrogen flux intake toward the crack tip neighborhood, slowing down the crack tip propagation rate $v_{c}$ as it is shown in the comparative analysis discussed in Fig. 5.

Fig. 5-a plots the crack tip distance $r_{f}$ as a function of time for both types of boundary conditions. While Fig. 5-b plots the crack tip propagation rates $v_{c}$ computed from the slope of the curves $r_{f}(t)$. It can be observed that $v_{c}$ determined with the boundary condition
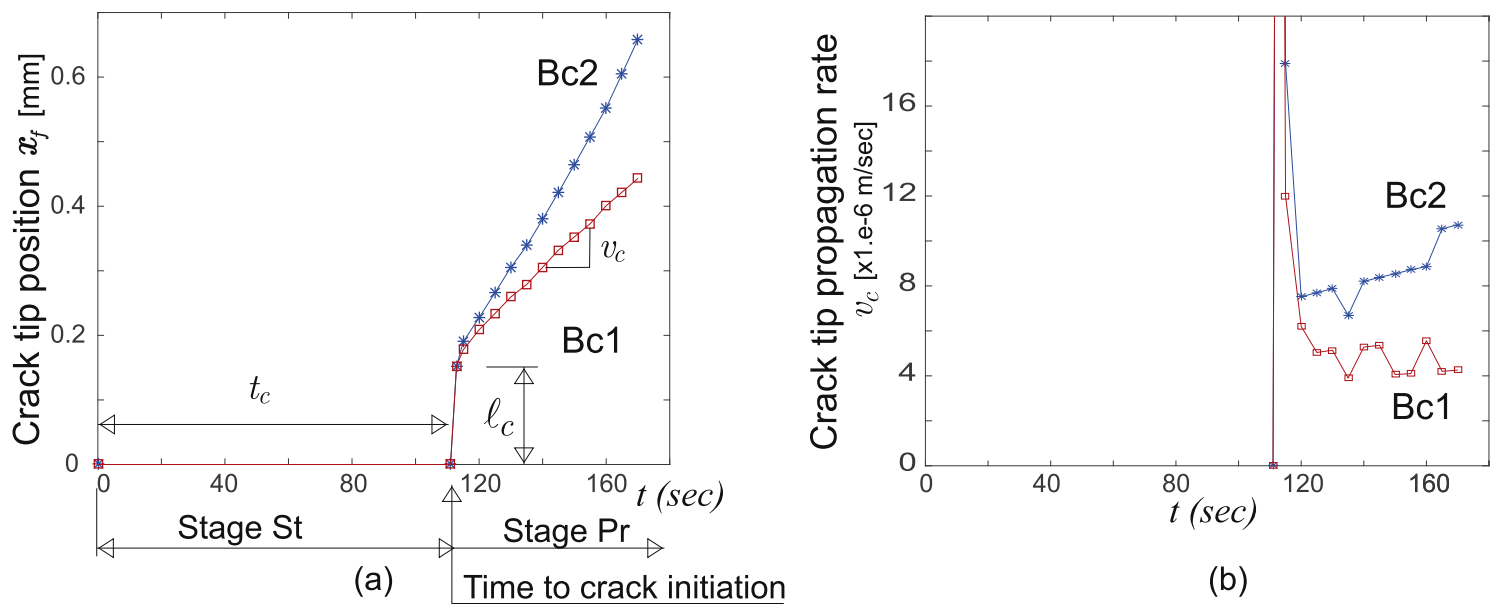

(b)

Fig. 5. Steel with $\sigma_{y}=1400 \mathrm{MPa}, K_{I}=50 \mathrm{MPa} \sqrt{m}, p=1.4$. a) Time vs. crack tip distance measured from the original notch tip for both hydrogen flux boundary conditions (Bc1 and Bc2, respectively). Stationary and propagating stages (Stage St and Pr) are depicted; b) time vs. crack tip propagation rate. 

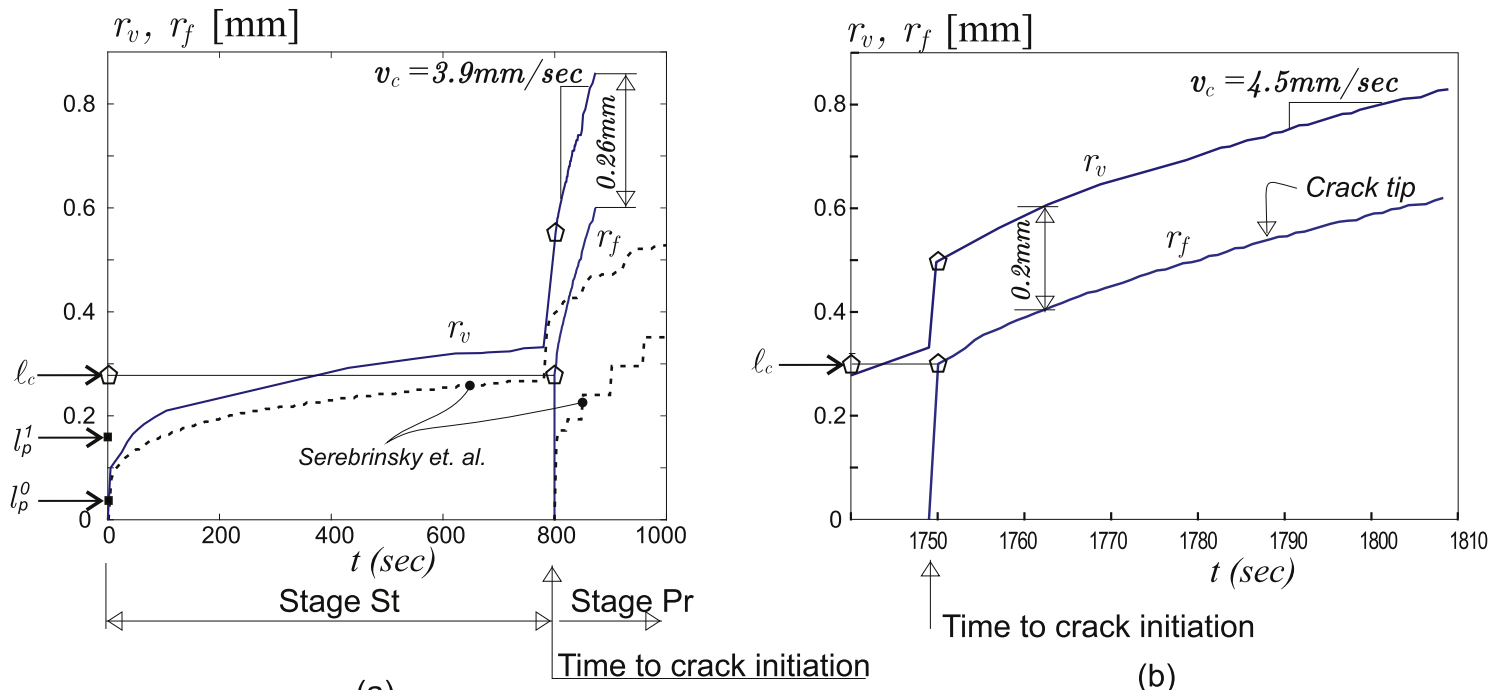

(a)

Time to crack initiation

(b)

Fig. 6. Crack tip distance $r_{f}$ and $r_{v}$ to the notch root, crack propagation rate, $v_{c}$ time to crack initiation, $t_{c}$, and fracture process zone size $\left(r_{v}-r_{f}\right)$ computed with: a) $l=50 \mu \mathrm{m}$; b) $l=30 \mu \mathrm{m}$. Simulated test: Steel B, $\sigma_{y}=1400 \mathrm{MPa}, p=1.4$ and boundary condition Bc2.

$\mathrm{Bc} 2$ is almost twice that computed with boundary condition Bc1. These results show the high sensitivity of $v_{c}$ with respect to the boundary condition imposing the hydrogen flux intake at the crack flanks.

4.1.4.3. Damaged zone size ahead of the crack tip. The role played by the parameter $l$ for governing different important variables in hydrogen-assisted fracture problems is of uppermost importance. To understand its role, we discuss several results corresponding to the simulation of Steel B, $\sigma_{y}=1400 \mathrm{MPa}, p=1.4$ and boundary condition Bc2 obtained with different values of $l$.

First, we recall that the ratio $\sigma_{c}^{P F} / \sigma_{y}$ depends primarily on $l$. So, from equation (73) and taking $l=30 \mu m$, the ratio $\sigma_{c}^{P F} / \sigma_{y}$ for steel B results $\sigma_{c}^{P F} / \sigma_{y}=3$, while taking $l=50 \mu \mathrm{m}$ it is $\sigma_{c}^{P F} / \sigma_{y}=2.3$.

Next, we analyze results in Fig. 6 . Time vs. distance $r_{f}$ of the point $x_{f}$ to the notch root, obtained with $l=50 \mu \mathrm{m}$ and $l=30 \mu \mathrm{m}$, are plotted in Fig. 6-a and b, respectively. The curves of time vs. distance $r_{v}$ are also plotted in the same Figures. We assume that the fracture process zone is characterized by those points having a damage value $0.05<\varphi \leq 0.97$ ahead of the crack tip. The fracture process zone sizes are shown in the Figure and remain almost constant during the crack propagation process. As expected, the fracture process zone size depends on $l$. Further, it increases with $l$ in a non-proportional manner. In Fig. 6-a, we also reproduce the results of crack tip distance $r_{f}$ and $r_{v}$ reported by the reference work (Serebrinksy et al.).

The variables $\ell_{c}, t_{c}$ and $v_{c}$ have also been computed and are depicted in Fig. 6, while $l_{p}^{0}$ and $l_{p}^{1}$ are shown in Fig. 6-a. It is observed a notorious dependence of the crack initiation time $t_{c}$ and the average of the crack tip propagation rate $v_{c}$ with the parameter $l$.

4.1.4.4. Hydrogen diffusion process. Fig. 7 displays the distributions of hydrogen concentration, cumulative plastic strain and damage in the crack tip zone at four different times, before and after crack initiation. Results correspond to a simulation of Steel B, load $K_{I}=50 \mathrm{MPa} \sqrt{m}$ and boundary condition Bc2. In this case, the crack initiation time is $t_{c}=800 \mathrm{sec}$.

Fig. 7-a shows the hydrogen distribution map at time $t=796 \mathrm{sec}$ which corresponds to a short time before the crack initiation. It is notable the increase of the local hydrogen concentration ahead of the notch tip which is caused by the gradients of the chemical potential induced by the high mean stresses. Hydrogen diffuses from distant areas toward the notch tip. The hydrogen concentration $c$ in the neighbor region of point A ranges between $10^{-5}$ and $1.2 \times 10^{-5}$, and the fracture energy is almost $20 \%$ of that corresponding to the pristine material. One plastic bulb is observed which is produced at the very beginning of the analysis. Its size is of the order of the plastic radius given by the formula: $R_{p} \approx 1 / 2 \pi\left(K_{I} / \sigma_{y}\right)^{2}=0.2 \mathrm{~mm}$ and after that, it remains unchanged.

At time $t_{c}=800 \mathrm{sec}$, Fig. 7-b, a crack initiates at point A and propagates in both directions, left and right. The initial crack propagation process is unstable, and the crack tip reaches almost instantaneously a length $\ell_{c}=0.285 \mathrm{~mm}$, measured from the notch root. The hydrogen concentration $c$ at point $x_{f}$ is $c=5 \times 10^{-6}$ and the fracture energy is $41 \%$ of the pristine material. For posterior times, $t>t_{c}$, the process stabilizes and the crack tip propagation rate reaches an almost constant value plotted in Fig. 6-a.

As the time increases, hydrogen is transported toward the inner domain, as shown in Fig. 7-c and d, a second plastic bulb initiates at point $\mathrm{B}(t=863 \mathrm{sec})$. It is not connected with the left one.

Fig. 8 displays the hydrogen concentrations at different times along a line coinciding with the horizontal symmetry plane of the plate. An abrupt diminution of concentration is observed between times $796 \mathrm{sec}$ and $800 \mathrm{sec}$ in the interval $0 \leq x \leq 0$. $285 \mathrm{~mm}$. It coincides with the crack initiation time, when the Bc2 is instantaneously imposed at the newly generated crack flanks.

At posterior times, the concentration profile ahead of the crack tip remains self-similar. 


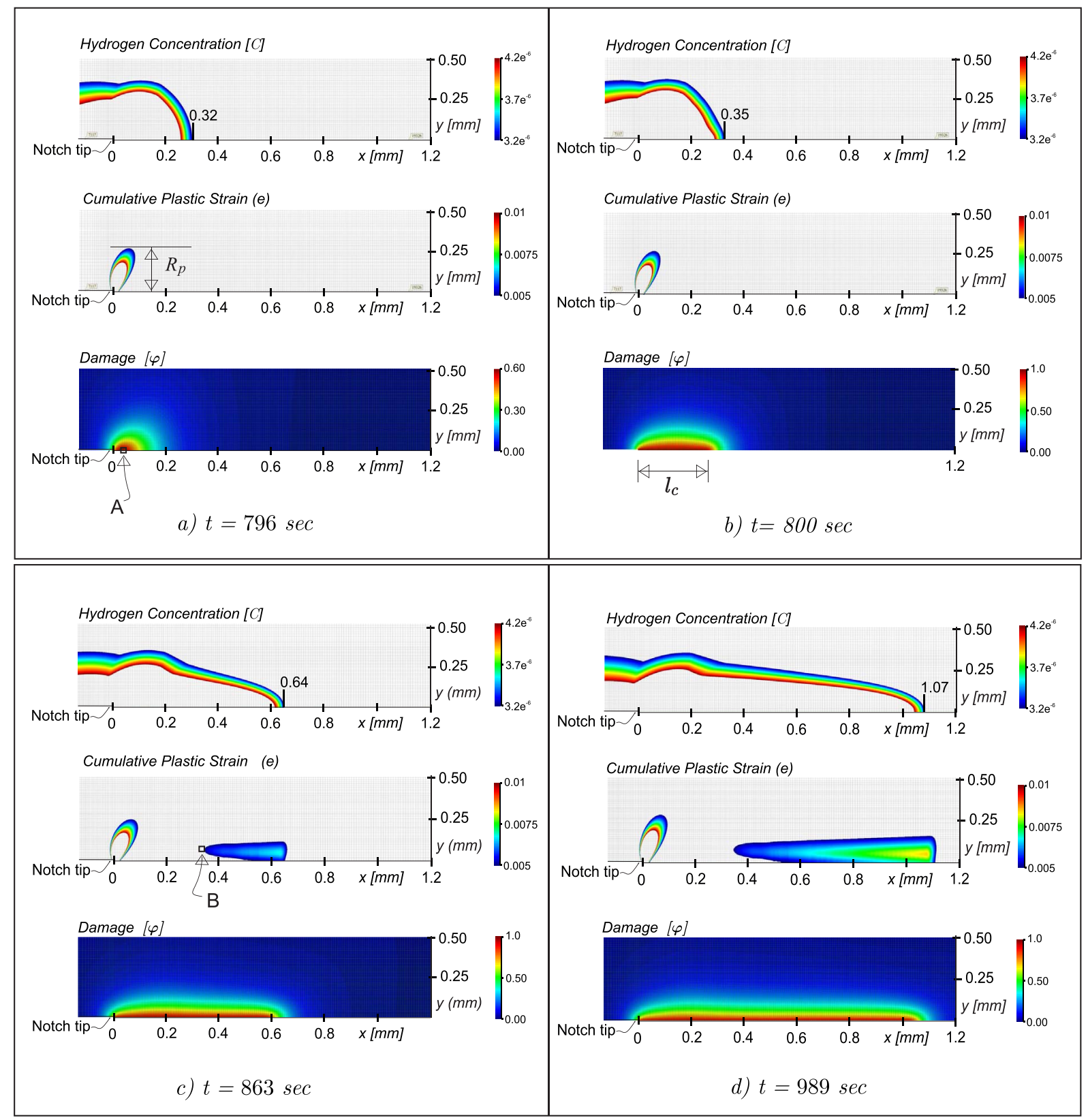

Fig. 7. Center sharp-notched panel test. Steel B, load $K_{I}=50 \mathrm{MPa} \sqrt{m}$, boundary condition Bc2. Color maps of hydrogen concentration, cumulative plastic strain and damage at times a) $t=798 \mathrm{sec}$; b) $t=t_{c}=800 \mathrm{sec}$; c) $t=863 \mathrm{sec}$ and d) $t=989 \mathrm{sec}$. (For interpretation of the references to colour in this figure legend, the reader is referred to the Web version of this article.)

Fig. 9 displays three deformed configurations at different times, before and after crack initiation. Around the crack flanks, it can be observed a well-defined region where strains localize.

4.1.4.5. Computed time to crack initiation. Fig. 10 displays the computed time to crack initiation for Steels A, B, C and several load levels. Also, in the same Figure are reported the results presented by Serebrinsky et al., as well as an experimental work (HM) taken from the same reference work.

Steels B and C have been simulated with a phase field characteristic length $l=50 \mu \mathrm{m}$, while Steel A has been simulated with two characteristic lengths: $l=150 \mu \mathrm{m}$ and $l=200 \mu \mathrm{m}$. By defining $l=150 \mu \mathrm{m}$ jointly with the material data in Table 1 , the ratio $\sigma_{c}^{P F} / \sigma_{y}$ results in $\sigma_{c}^{P F} / \sigma_{y}=2.3$, while for the length $l=200 \mu \mathrm{m}$, this ratio is $\sigma_{c}^{P F} / \sigma_{y}=1.8$. 


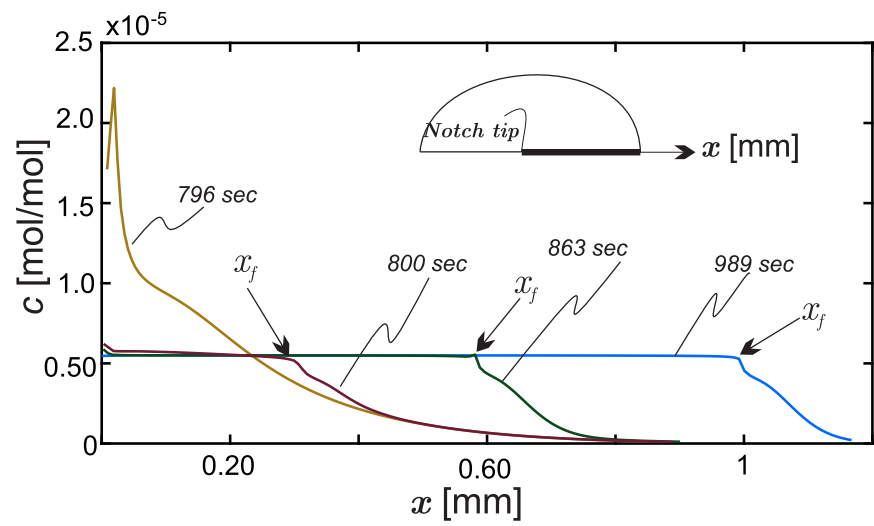

Fig. 8. Hydrogen bulk concentration, given in mol of atomic hydrogen per mol of solvent, along the crack line.

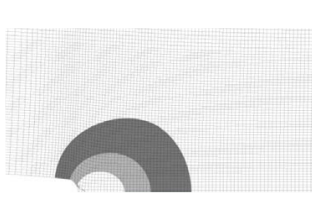

a) $t=798 \mathrm{sec}$

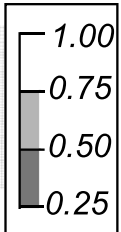

0.25

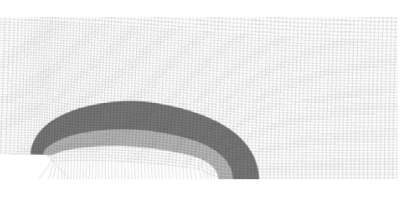

b) $t=800 \mathrm{sec}$

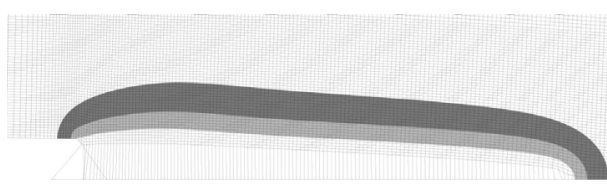

c) $t=950 \mathrm{sec}$

Fig. 9. Deformed configurations at different times and phase field distribution. Steel B, load $K_{I}=50 \mathrm{MPa} \sqrt{m}$, boundary condition Bc2.

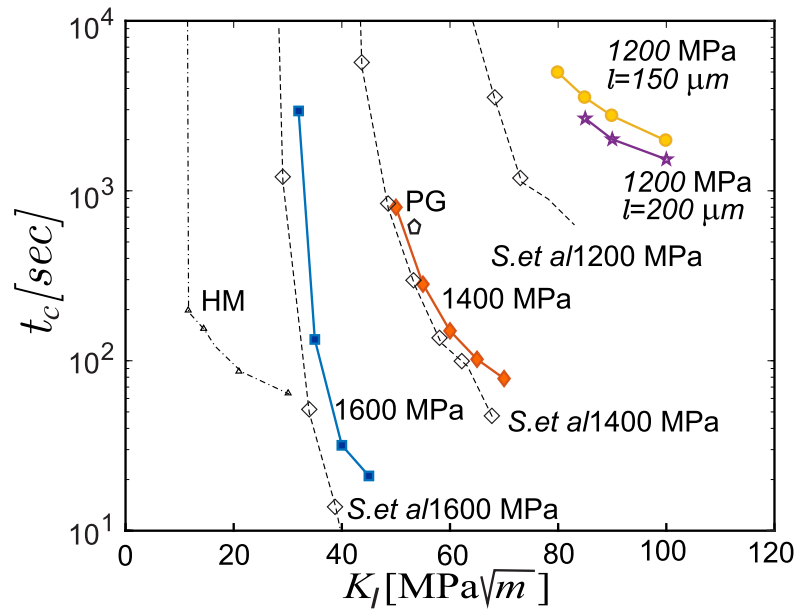

Fig. 10. Time to crack initiation vs. load for different high strength steels. Curves with filled symbols correspond to present results. Curves (S-1200 MPa, S-1400 MPa and S-1600 MPa) with hollow symbols correspond to the reference work of Serebrinsky et al. HM experimental (taken from Hirose and Mura, 1984a), steel with $\sigma_{y}=1530 \mathrm{MPa}$ and notch $\operatorname{root} \rho=0.25 \mathrm{~mm}$ ).

All steels show a stress intensity threshold value, $K_{S C C}$, in the sense that for enough low loads, with $K_{I}<K_{S C C}$, the time to crack initiation $t_{c}$ tends to $t_{c} \rightarrow \infty$. Therefore, no crack is observed for the assumed environment and load below $K_{S C C}$. We remark that the values of $K_{S C C}$ obtained with the present model remain within the range of reported values in experimental works.

4.1.4.6. Crack tip propagation rate for different load levels. Fig. 11 plots the load vs. crack tip propagation rate curves obtained for Steel $\mathrm{B}$ and utilizing boundary condition $\mathrm{Bc} 2$. In the Figure, experimental results are also reproduced for similar steels which have been cited in Serebrinsky et al. It can be noted that the present results lay within the range of data reported for this parameter. In any way, a notable dispersion of experimental results in the literature is observed.

4.1.4.7. Dilatational strain effect caused by hydrogen concentration. Next, we study the effect produced by the dilatational strain $\mathbf{E}_{c}$ on the time to crack initiation. Dilatational strains caused by the presence of hydrogen in the lattice was analyzed by Lufrano et al. 


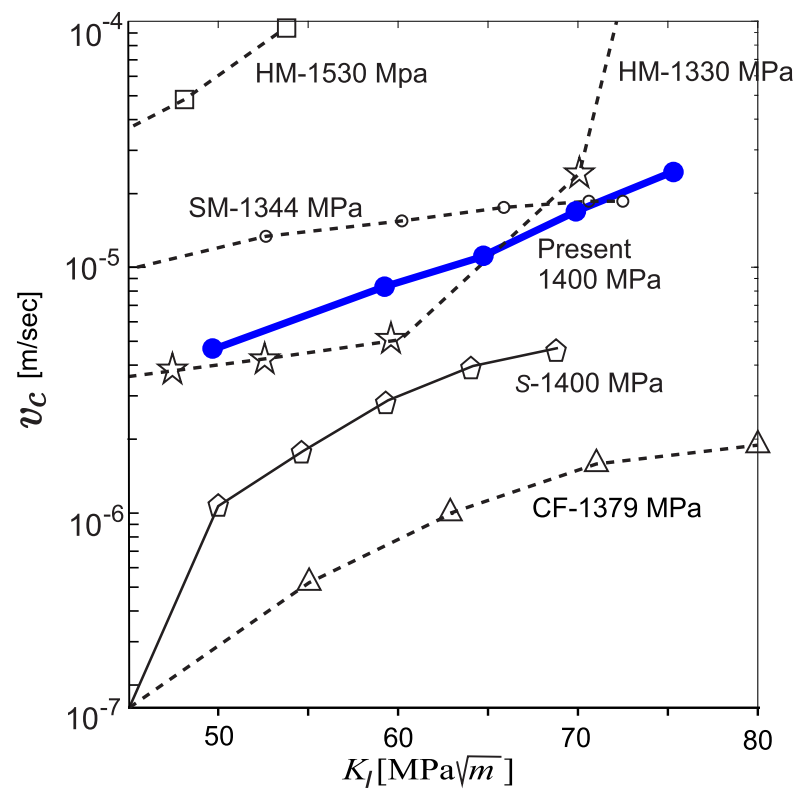

Fig. 11. Load vs. crack tip propagation rate. Present results for Steel B. Numerical solutions reported by Serebrinsky et al. are $S-1400 \mathrm{MPa}$. Experimental results reported by Hirose and Mura (1984b) are: HM-1530 MPa and HM-1330 MPa. Results SM-1344 MPa have been reported by Simmons et al. (1978) and CF-1379 MPa by Colangelo and Ferguson (1969).

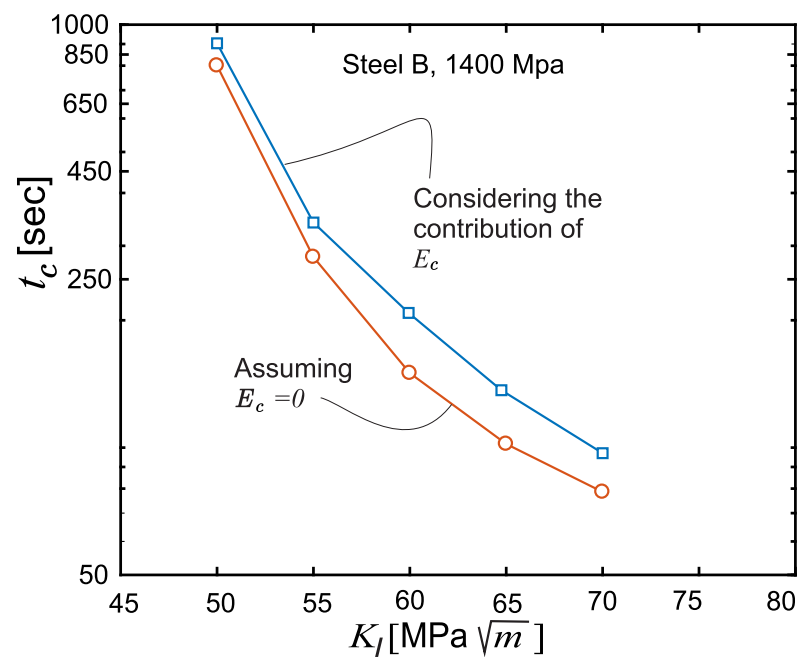

Fig. 12. Curves of crack time initiation versus load level for Steel B, with $\sigma_{y}=1400 \mathrm{MPa}$, considering, or not, the volume of trapped hydrogen

(1998).

When the hydrogen concentration increases at the crack tip zone, due to the stress-assisted diffusion process, the strain $\mathbf{E}_{c}$ introduces a compressive stress state inducing a stress relaxation effect, or shielding effect, for the damage evolution. Therefore, the crack initiation time is delayed.

This response is observed in Fig. 12. Results taking into account $\mathbf{E}_{c}$ display a notable increase of time to crack initiation for plates subjected to identical loads if compared with those obtained by neglecting $\mathbf{E}_{c}$.

\section{Conclusion}

This paper presents two principal contributions:

- a novel thermodynamical-based formulation of elastic-plastic deformation, phase-field fracture, and solute migration in solids;

- one application of the model to simulate the hydrogen assisted cracking problem of a sustained load test and different steels. 
We have not intended to rigorously reproduce experimental data, but to investigate the model capacity to simulate subcritical crack growth using the general thermodynamical formulation described in the first part of the paper.

Results obtained with the phase field model qualitatively agree with those described in previous numerical works. The model reproduces the experimental trends observed for some of the most important variables involved in the time-dependent subcritical cracking problem assisted by the environment, such as $t_{c}, v_{c}$, and $\ell_{c}$, and their sensitivities to material properties, load levels, impurity concentration, etc. The model can explain the general trends of widely observed experimental results relating the velocity versus stress intensity factor $\left(v_{c}-K_{I}\right)$ curves. Specifically, those stages denoted as I, II, and III in the literature.

An important aspect of the model has been the choice of the degradation law (75), taken from the literature and modified with the parameter $p$ to capture the sensitivity of variables $t_{c}, v_{c}$ and $\ell_{c}$ with the yield stress, plastic hardening exponent and fracture toughness has been calculated with the same degradation model. The exponent $p$, in the law (75), has been adjusted for one test (Steel B and $K_{I}=50 \mathrm{MPa} \sqrt{m}$ ). Then, the so-adjusted law has been utilized for computing the dependence of $t_{c}$ and stages denoted St and Pr, with different steels and load conditions.

In addition to the couplings between plastic deformation and solute diffusion discussed in this paper, other couplings are wellknown to be important. For instance, solute hydrogen atoms can be trapped at and transported by dislocations generated by plastic deformation, see Dadfarnia et al.(2015). To account for these facts, one could generalize the theory presented here by considering the presence of two types of interconvertible solute species, one that occupies normal interstitial lattice sites and other that occupies trapping sites associated with dislocations as done in Di Leo and Anand (2013). The mentioned couplings between plastic deformation and solute diffusion can be taken into account by considering that: the chemical potential of the trapped species depends on the plastic deformation since the number of trapping sites does (Di Leo and Anand, 2013); the flux of the trapped species depends on the rate of plastic strain (Dadfarnia et al., 2015). These topics will be investigated in the future.

\section{Acknowledgments}

The first author gratefully acknowledges financial support provided by the Brazilian agencies, CNPq (311587/2016-0) and FAPERJ (E-26/110.037/2014).

The fourth author gratefully acknowledges financial support provided by ANPCyT (PICT 2014-3372), Argentina.

The research leading to these results has received funding from the European Research Council under the European Union's Seventh Framework Programme (FP/2007-2013)/ERC Grant Agreement 320815 (ERC Advanced Grant Project "Advanced tools for computational design of engineering materials-COMP-DES-MAT").

The Joint Research Project Mercosur PPCP 004-2011 (CAPES, Brazil, SPU, Argentina) and the Bilateral Program FAPERJ/ CONICET have also provided additional financial support.

\section{Appendix I. Variational form of the initial boundary value problem and numerical model}

Equations (69)-(72), supplemented with appropriate initial and boundary conditions constitute an initial-boundary value problem (IBVP) for the fields $\mathbf{u}, c, e$ and $\varphi$. In this Appendix, we introduce a variational form of the IBVP which is amenable to numerical treatment via the finite-element method. The variational form can be obtained directly from a modification of the principle of virtual power as discussed in what follows.

Let us consider the body $\mathcal{B}$, at some fixed time $t$, subjected to the following boundary conditions:

- Mechanical boundary conditions. The traction $\mathbf{s}$ and displacement $\overline{\mathbf{u}}$ are prescribed functions on the complementary surfaces $\partial \mathcal{B}_{S}$ and $\partial \mathcal{B}_{u}$ of $\partial \mathcal{B}$, that is

$$
\mathbf{S n}=\mathbf{s} \quad \text { on } \partial \mathcal{B}_{S}, \quad \mathbf{u}=\overline{\mathbf{u}} \text { on } \partial \mathcal{B}_{u}
$$

- Chemical boundary conditions. Impermeability and local chemical equilibrium prevail on the complementary surfaces $\partial \mathcal{B}_{h}$ and $\partial \mathcal{B}_{c}$ of $\partial \mathcal{B}$, that is

$$
\mathbf{h} \cdot \mathbf{n}=0 \quad \text { on } \partial \mathcal{B}_{h}, \quad \mu=\mu_{\text {env }} \text { on } \partial \mathcal{B}_{c},
$$

where $\mu_{\mathrm{env}}$, the chemical potential of atomic hydrogen in the environment, is a prescribed function on $\partial \mathcal{B}_{c}$;

- Phase-field boundary condition. The null microtraction condition holds on the complete boundary $\partial \mathcal{B}$, that is

$$
\xi \cdot \mathbf{n}=0 \quad \text { on } \quad \partial \mathcal{B}
$$

Here, $\mathbf{n}$ is the outward unit normal vector field to $\partial \mathcal{B}$. Notice that $(70)_{3}$ and $(\mathrm{AI}-2)_{2}$ yield a non-linear boundary condition for the field $c$,

$$
c=\bar{c}\left(\varphi, \nabla \varphi, c, \sigma_{\mathrm{m}}\right) \text { on } \partial \mathcal{B}_{c},
$$

where: 


$$
\bar{c}\left(\varphi, \nabla \varphi, c, \sigma_{m}\right)=c_{e q} \exp \left(-\frac{\mu_{\sigma}\left(\sigma_{m}\right)+\mu_{f}(\varphi, c)}{R T}\right) .
$$

and $c_{e q}$ is the hydrogen concentration in equilibrium with environment in absence of stress and damage. Initial conditions could be defined through: $\varphi=0$ and $c=c_{0}$ on $\mathcal{B}$.

For the sake of numerical treatment, we follow Miehe et al. (2010) and assume that (72) can be replaced by

$$
2(1-\varphi) \mathcal{H}-\frac{1}{l}\left(g_{f}(c) \varphi-l^{2} \operatorname{Div}\left(g_{f}(c) \nabla \varphi\right)\right)=0,
$$

with "the local history field of maximum reference energy" $\mathcal{H}$ satisfying

$$
\dot{\mathscr{H}}=\left\{\begin{array}{cc}
\dot{\psi}_{e}^{0}\left(\mathbf{E}_{e}\right) & \text { if } \dot{\psi}_{e}>0, \\
0 & \text { otherwise. }
\end{array}\right.
$$

This assumption was also adopted by the authors in Duda et al. (2015). An alternative driving force term, motivated by the discussion presented by Miehe et al. (2015), has also been tested in the present numerical model and is given by

$$
\dot{\mathscr{H}}=\left\{\begin{array}{cc}
\frac{1}{2} \frac{\sqrt{\langle\mathbf{S}}\rangle: \mathbf{E}_{e}}{} & \text { if: } \overline{\langle\widetilde{\mathbf{S}}\rangle: \mathbf{E}_{e}}>0 \\
0 & \text { otherwise }
\end{array} \quad ; \quad\langle\widetilde{\mathbf{S}}\rangle=\sum_{i=1}^{3}\left\langle\widetilde{S}_{i}\right\rangle \mathbf{e}_{i} \otimes \mathbf{e}_{i},\right.
$$

where $\widetilde{\mathbf{S}}=\partial \psi_{e}^{0} / \partial \mathbf{E}_{\mathrm{e}}$ is the effective stress and (AI-8) $)_{2}$ identifies the tensile part of this tensor. The driving force term (AI-8) freezes the damage evolution in compressive stress regimes which could arise in regions close to the crack tip due to the effect produced by the strain $\mathbf{E}_{c}$. Note that in tensile stress regimes, the driving forces (AI-7) and (AI-8) are identical.

Next, let us consider the following functional spaces defined on $\mathcal{B}$

$$
\begin{aligned}
& \mathscr{U}^{u}=\left\{\mathbf{u} \in \mathbf{H}^{1}(\mathscr{B}) \mid \mathbf{u}=\overline{\mathbf{u}} \quad \text { on } \quad \partial \mathscr{B}_{\mathbf{u}}\right\} ; \\
& \mathscr{U}^{\varphi}=\left\{\varphi \in \mathbf{H}^{1}(\mathscr{B})\right\} ; \\
& \mathscr{U}^{c}=\left\{c \in \mathbf{H}^{1}(\mathscr{B}) \mid c=\bar{c} \quad \text { on } \quad \partial \mathscr{B}_{c}\right\} .
\end{aligned}
$$

with $\mathbf{H}^{1}$ being a space of smooth enough functions. $\mathscr{U}^{u}$ is the space of admissible displacements, $\mathscr{U}^{\varphi}$ the space of admissible phasefields and $\mathscr{U}^{c}$ is the space of admissible hydrogen concentrations. Their corresponding admissible spaces of variations are

$$
\begin{aligned}
& \mathscr{V}^{u}=\left\{\widetilde{\mathbf{u}} \in \mathbf{H}^{1} \mid \widetilde{\mathbf{u}}=0 \quad \text { on } \quad \partial \mathcal{B}_{\mathbf{u}}\right\} ; \\
& \mathscr{V}^{\varphi}=\mathscr{U}^{\varphi} \\
& \mathscr{V}^{\alpha}=\left\{\tilde{\alpha} \in \mathbf{H}^{1} \mid \tilde{\alpha}=0 \quad \text { on } \quad \partial \mathcal{B}_{\mathbf{c}}\right\} ;
\end{aligned}
$$

Then, the variational problem consists of finding the functions $\mathbf{u} \in \mathscr{V}^{u}, \varphi \in \mathscr{V}^{\varphi}$, and $c \in \mathscr{V}^{c}$ such that they fulfill the following equations:

$$
\begin{aligned}
& \int_{\mathcal{B}} \mathbf{S} \cdot \nabla^{s} \widetilde{\mathbf{u}} \mathrm{d} V-\int_{\mathcal{B}} \mathbf{b} \cdot \widetilde{\mathbf{u}} \mathrm{d} V-\int_{\partial \mathcal{B}_{S}} \mathbf{s} \cdot \widetilde{\mathbf{u}} \mathrm{d} A=0 ; \quad \forall \widetilde{\mathbf{u}} \in \mathscr{V}^{u} \\
& \int_{\mathcal{B}} \lg _{f}(\nabla \varphi \cdot \nabla \widetilde{\varphi}) \mathrm{d} V+\int_{\mathcal{B}}\left(-2(1-\varphi) \mathcal{H}+\frac{g_{f}}{l} \varphi\right) \widetilde{\varphi} \mathrm{d} V=0 ; \quad \forall \widetilde{\varphi} \in \mathscr{V}^{\varphi} \\
& \int_{\mathcal{B}} \dot{c} \tilde{\alpha} \mathrm{d} V+\int_{\mathcal{B}} M c \nabla \mu \cdot \nabla \widetilde{\alpha} \mathrm{d} V=0 ; \quad \forall \tilde{\alpha} \in \mathscr{V}^{\alpha}
\end{aligned}
$$

where the chemical potential gradient, $\nabla \mu(\mathbf{x}, t)$, is evaluated as defined in next sub-Section.

\section{Finite element implementation}

We propose a four-node quadrilateral finite element interpolation including the following fields: displacement $\mathbf{u}$, phase-field $\varphi$ and hydrogen concentration $c$. All these fields are interpolated using bilinear functions satisfying

$$
\mathbf{u}(\mathbf{x}, t)=\sum_{i=1}^{n_{\text {nod }}} N_{i}(\mathbf{x}) \hat{\mathbf{u}}_{i}(t) \quad ; \quad \varphi(\mathbf{x}, t)=\sum_{i=1}^{n_{\text {nod }}} N_{i}(\mathbf{x}) \hat{\varphi}_{i}(t) \quad ; \quad c(\mathbf{x}, t)=\sum_{i=1}^{n_{\text {nod }}} N_{i}(\mathbf{x}) \hat{c}_{i}(t)
$$

with $n_{\text {nod }}$ being the number of nodes of the finite element mesh, $N_{i}$ is the shape function associated with the $i-t h$ node and $\hat{\mathbf{u}}_{i}, \hat{\varphi}_{i}$ and $\hat{c}_{i}$ are the nodal interpolation parameters of the respective fields. Nodal parameters are identified with the symbol $(\hat{*})$. The corresponding admissible variations are also defined as follows

$$
\widetilde{\mathbf{u}}(\mathbf{x})=\sum_{i=1}^{n_{\text {nod }}} N_{i}(\mathbf{x}) \hat{\widetilde{\mathbf{u}}}_{i} \quad ; \quad \tilde{\varphi}(\mathbf{x})=\sum_{i=1}^{n_{\text {nod }}} N_{i}(\mathbf{x}) \hat{\tilde{\varphi}}_{i} \quad ; \quad \tilde{\alpha}(\mathbf{x})=\sum_{i=1}^{n_{\text {nod }}} N_{i}(\mathbf{x}) \hat{\tilde{\alpha}}_{i} .
$$

In a generic finite element $\mathfrak{e}$, the strain $\mathbf{E}$ and the admissible variation of strain $\widetilde{\mathbf{E}}$ are given as follows: 


$$
\mathbf{E}(\mathbf{x})=\mathbb{B}^{\mathfrak{e}}(\mathbf{x}) \hat{\mathbf{u}}^{\mathfrak{e}} \quad \widetilde{\mathbf{E}}(\mathbf{x})=\mathbb{B}^{\mathfrak{e}}(\mathbf{x}) \hat{\widetilde{\mathbf{u}}}^{\mathfrak{e}}
$$

where $\hat{\mathbf{u}}^{\mathfrak{e}}$ is the nodal displacement vector of the element $\mathfrak{e}$ and the strain-displacement matrix $\mathbb{B}^{\mathfrak{e}}$ is defined according with the $\mathrm{B}$-Bar technique (see Duda et al., 2015). In consonance with the B-Bar technique and to avoid numerical instabilities, the interpolation of the strain component $\mathbf{E}_{c}(\mathbf{x})$ must be approached with an element-wise constant interpolation.

Considering (70), the chemical potential field $\mu(\mathbf{x}, t)$ in (AI-13), is approached using the following interpolation

$$
\mu(\mathbf{x}, t)=\mu_{0}+R T \ln c(\mathbf{x}, t)+\mu_{f}^{h}(\mathbf{x}, t)+\mu_{\sigma}^{h}(\mathbf{x}, t)
$$

where $c(\mathbf{x}, t)$ is given by (AI-14) and:

$$
\mu_{f}^{h}(\mathbf{x}, t)=\sum_{i=1}^{n_{\text {nod }}} N_{i}(\mathbf{x})\left(\hat{\mu}_{f}\right)_{i}(t) \quad ; \quad \mu_{\sigma}^{h}(\mathbf{x}, t)=-\sum_{i=1}^{n_{\text {nod }}} N_{i}(\mathbf{x})\left(\hat{\mu}_{\sigma}\right)_{i}(t)
$$

with the nodal values, $\left(\hat{\mu}_{f}\right)_{i}$ and $\left(\hat{\mu}_{\sigma}\right)_{i}$, being obtained by a conventional smoothing of the respective terms $\mu_{f}$ and $\mu_{\sigma}$ known at the quadrature points and according to expression (70).

\section{Discrete equations of the problem}

Substituting equations (AI-14)-(AI-18) into the variational problem (AI-11)-(AI-13), yields:

- Force balance:

$$
\mathbb{R}_{\mathbf{S}}(\mathbf{u}, e, \varphi, c)=\Lambda_{\mathfrak{e}=1}^{n_{\text {elem }}} \int_{\mathcal{B}^{\mathfrak{e}}}\left(\mathbb{B}^{\mathfrak{e}}\right)^{T} \mathbf{S} \mathrm{d} V-\mathbb{F}_{u}^{\text {ext }}=0 ;
$$

where $\Lambda$ is the finite element assembling operator, $n_{\text {elem }}$ is the number of finite elements in the mesh and $\mathbf{S}$ is the stress term defined by the constitutive model. The vector $F_{u}^{\text {ext }}$ is derived from the second and third terms in (AI-11) and represents the conventional external forces.

- Damage equation:

$$
\mathbb{R}_{\varphi}(\mathbf{u}, e, \varphi, c)=\left[\mathbb{K}_{\varphi}+\mathbb{M}_{\varphi}\right][\hat{\varphi}]-\mathbb{F}_{\varphi}^{\mathrm{ext}}=0 ;
$$

where $[\hat{\varphi}]$ is the global vector of interpolation parameters (collecting all the finite element mesh parameters) of $\varphi$, and:

$$
\begin{aligned}
& \mathbb{K}_{\varphi}=\Lambda_{\mathfrak{e}=1}^{n_{e} l e m} \mathbb{K}_{\varphi}^{\mathfrak{e}} ; \quad\left[\mathbb{K}_{\varphi}^{\mathfrak{e}}\right]_{i, j}=\int_{\mathscr{B}^{\mathfrak{e}}} \lg _{f}\left(\left(\nabla N_{i}^{\mathfrak{e}}\right)^{T} \cdot \nabla N_{j}^{\mathfrak{e}}\right) \mathrm{d} V ; \quad\left[\nabla N_{i}^{\mathfrak{e}}\right]=\left[\begin{array}{c}
N_{i, x}^{\mathfrak{e}} \\
N_{i, y}^{\mathfrak{e}}
\end{array}\right] ; \\
& \mathbb{M}_{\varphi}=\bigwedge_{\mathfrak{e}=1}^{n_{\text {elem }}} \mathbf{M}_{\varphi}^{\mathfrak{e}} ; \quad\left[\mathbf{M}_{\varphi}^{\mathfrak{e}}\right]_{i, j}=\int_{\mathscr{B}}\left(2 \mathscr{H}+\frac{g_{f}}{l}\right)\left(N_{i}^{\mathfrak{e}} N_{j}^{\mathfrak{e}}\right) \mathrm{d} V ; \\
& \mathbb{F}_{\varphi}^{\text {ext }}=\Lambda_{\mathfrak{e}=1}^{n_{\text {elem }}}\left(\mathbb{F}_{\varphi}^{\mathfrak{e}}\right)^{\text {ext }} ; \quad\left[\left(\mathbb{F}_{\varphi}^{\mathfrak{e}}\right)^{\text {ext }}\right]_{i}=\int_{\mathscr{B}^{\mathfrak{e}}} 2 \mathscr{H} N_{i}^{\mathfrak{e}} \mathrm{d} V .
\end{aligned}
$$

- Hydrogen diffusion equation:

$$
\mathbb{R}_{c}(\mathbf{u}, e, \varphi, c)=\mathbb{M}_{c}[\dot{\hat{c}}]+\left(\mathbb{K}_{c}+\mathbb{G}_{c}\right)[\hat{c}]=0
$$

where $[\hat{c}]$ is the global vector of interpolation parameters of $c$, and:

$$
\begin{aligned}
& \mathbb{M}_{c}=\bigwedge_{\mathfrak{e}=1}^{n_{\text {elem }}} \mathbb{M}_{c}^{\mathfrak{e}} ; \quad\left[\mathbb{M}_{c}^{\mathfrak{e}}\right]_{i, j}=\int_{\mathscr{B}^{\mathfrak{e}}}\left(N_{i}^{\mathfrak{e}} N_{j}^{\mathfrak{e}}\right) \mathrm{d} V ; \\
& \mathbb{K}_{c}=\bigwedge_{\mathfrak{e}=1}^{n_{\text {elem }}} \mathbb{K}_{c}^{\mathfrak{e}} ; \quad\left[\mathbb{K}_{c}^{\mathfrak{e}}\right]_{i, j}=\int_{\mathscr{B}^{\mathfrak{e}}} M R T\left(\left(\nabla N_{i}^{\mathfrak{e}}\right)^{T} \cdot \nabla N_{j}^{\mathfrak{e}}\right) \mathrm{d} V ; \\
& \mathbb{G}_{c}\left(c, \varphi, \sigma_{m}\right)=\bigwedge_{\mathfrak{e}=1}^{n_{\text {elem }}} \mathbb{G}_{c}^{\mathfrak{e}}\left(c, \varphi, \sigma_{m}\right) ; \quad\left[\mathbb{G}_{c}^{\mathfrak{e}}\right]_{i, j}=\int_{\mathscr{B}^{\mathfrak{e}}} M\left[\left(\nabla N_{i}^{\mathfrak{e}}\right)^{T} \cdot \nabla\left(\mu_{f}+\mu_{\sigma}\right)^{\mathfrak{e}}\right] N_{j}^{\mathfrak{e}} \mathrm{d} V .
\end{aligned}
$$

\section{Time integration scheme}

Equations (AI-19), (AI-20) and (AI-22), are solved using a staggered time integration scheme that is described in Box 1. The residues of those equations are denoted in the Box by $\mathbf{R}_{\mathbf{S}}, \mathbf{R}_{\varphi}$ and $\mathbf{R}_{c}$, respectively. Subindices $n$ and $n+1$ indicate the values of the functions at two successive time steps. Equation (AI-26) is derived from (AI-22) by applying an Euler-backward time integration scheme to integrate the hydrogen concentration.

By solving equation (AI-24), Box 1, in the first step of this staggered scheme, the incremental inequality $\Delta e \geq 0$ is pointwise guaranteed by means of a conventional return mapping algorithm. 


\section{Box 1}

Staggered time integration scheme for solving the coupled elasto-plastic-phase field problem with solute diffusion.

LOOP of time steps: $(n+1)$

Given $\mathbf{u}_{n}, e_{n}, \varphi_{n}, c_{n}$ and defining: $\varphi_{n+1}^{0}=\varphi_{n}$;

Find: $\mathbf{u}_{n+1}, e_{n+1}, \varphi_{n+1}$ and $c_{n+1}$ as follows:

LOOP of mechanical iterations $(i)$. Do while $\left\|\varphi_{n+1}^{i}-\varphi_{n+1}^{i-1}\right\|_{\infty}>\operatorname{tol}_{\varphi}$

1) Using a Newton-Raphson scheme, find $\mathbf{u}_{n+1}^{i}$ and $e_{n+1}^{i}$ by solving:

$$
\mathbb{R}_{\mathbf{S}}\left(\mathbf{u}_{n+1}^{i}, e_{n+1}^{i}, \varphi_{n+1}^{i-1}, c_{n}\right)=0
$$

2) Find $\varphi_{n+1}^{i}$ by solving the linear equation system:

$$
\mathbb{R}_{\varphi}\left(\mathbf{u}_{n+1}^{i}, e_{n+1}^{i}, \varphi_{n+1}^{i}, c_{n}\right)=0 ;
$$

END LOOP of mechanical iterations.

3) With the mechanical LOOP solution $\left(\mathbf{u}_{n+1} ; e_{n+1} ; \varphi_{n+1}\right)$, find $c_{n+1}$ by solving:

$$
\mathbb{R}_{c}\left(\mathbf{u}_{n+1}, e_{n+1}, \varphi_{n+1}, c_{n+1}\right)=0 ;
$$

END LOOP of time steps.

Also, following an identical procedure proposed in Duda et al. (2015), by solving equation (AI-25) in the second step of the staggered scheme, the inequality $\Delta \varphi(\mathbf{x})=\varphi_{n+1}(\mathbf{x})-\varphi_{n}(\mathbf{x}) \geq 0$ in $\mathcal{B}$ is satisfied by defining the compact history field $\mathcal{H}\left(\mathbf{E}_{e}, t_{n+1}\right)$ as follows

$$
\begin{array}{lll}
\mathscr{H}\left(\mathbf{x}, t_{n+1}\right)=\psi_{e}\left(\mathbf{E}_{e}\left(\mathbf{x}, t_{n+1}\right)\right) & \text { if } & \psi_{e}\left(\mathbf{E}_{e}\left(\mathbf{x}, t_{n+1}\right)\right)>\psi_{e}\left(\mathbf{E}_{e}\left(\mathbf{x}, t_{n}\right)\right) ; \\
\mathscr{H}\left(\mathbf{x}, t_{n+1}\right)=\mathscr{H}\left(\mathbf{x}, t_{n}\right) & \text { if } & \psi_{e}\left(\mathbf{E}_{e}\left(\mathbf{x}, t_{n+1}\right)\right) \leq \psi_{e}\left(\mathbf{E}_{e}\left(\mathbf{x}, t_{n}\right)\right) .
\end{array}
$$

An identical treatment is utilized when the alternative driving force (AI-8) is adopted instead of (AI-7). Thus, the function $\mathcal{H}$

$$
\mathcal{H}\left(\mathbf{x}, t_{n+1}\right) \geq \mathcal{H}\left(\mathbf{x}, t_{n}\right)
$$

for every time in $\mathcal{B}$. In Duda et al. (2015), it has been shown that using this technique, the inequality $\Delta \varphi(\mathbf{x}) \geq 0$ is satisfied.

The term $\mu_{f}(\varphi, c)$ and $\mu_{\sigma}\left(\sigma_{m}, c\right)$ in matrix $\mathbb{G}_{c}$ of equation (AI-23), is computed with the concentration $c_{n}$ of the previous time step. Then, equation (AI-26) is linear in the vector $[\hat{c}]$.

\section{Appendix A. Supplementary data}

Supplementary data related to this article can be found at http://dx.doi.org/10.1016/j.ijplas.2017.11.004.

\section{References}

Aifantis, E.C., 1987. The physics of plastic deformation. Int. J. Plasticity 3 (3), 211-247.

Anderson, T.L., 2005. Fracture Mechanics: Fundamentals and Applications. CRC Press.

Baierlein, R., 2001. The elusive chemical potential. Am. J. Phys. 69 (4), 423-434.

Capriz, G., 1989. Continua with Microstructure. Springer.

Colangelo, V.J., Ferguson, M.S., 1969. The role of the strain hardening exponent in stress corrosion cracking of a high strength steel. Corrosion 25 (12), $509-514$. Coleman, B.D., Noll, W., 1963. The thermodynamics of elastic materials with heat conduction and viscosity. Archive Ration. Mech. Analysis 13, 167-178. da Silva, M.N., Duda, F.P., Fried, E., 2013. Sharp-crack limit of a phase-field model for brittle fracture. J. Mech. Phys. Solids 61 (11), $2178-2195$.

Dadfarnia, M., Schembri, P.E., Sofronis, P., Foulk, J.W., Nibur, K.A., Balch, D.K., et al., 2014. On modeling hydrogen-induced crack propagation under sustained load. JOM 66 (8), 1390-1398.

Dadfarnia, M., Martin, M.L., Nagao, A., Sofronis, P., Robertson, I.M., 2015. Modeling hydrogen transport by dislocations. J. Mech. Phys. Solid. 78, 511-525.

Di Leo, C.V., Anand, L., 2013. Hydrogen in metals: a coupled theory for species diffusion and large elastic-plastic deformations. Int. J. Plasticity 43, 42-69.

Duda, F.P., Souza, A.C., 2002. Continuum mechanics, inelastic behavior and multiscale modeling in mechanics of materials. Comput. Appl. Math. 21 (2), 445-498.

Duda, F.P., Barbosa, J.M., Guimarães, L.J., Souza, A.C., 2007. Modeling of coupled deformation-diffusion-damage in elastic solids. Int. J. Model. Simul. Petroleum Industry 1 (1)

Duda, F.P., Souza, A.C., Barbosa, J.M., Guimarães, L.J., 2009. A one-dimensional theory of solute diffusion and degradation in elastic solids. J. Elast. 97 (1), 15-30. Duda, F.P., Souza, A.C., Fried, E., 2010. A theory for species migration in a finitely strained solid with application to polymer network swelling. J. Mech. Phys. Solids 58 (4), 515-529.

Duda, F.P., Ciarbonetti, A., Sánchez, P.J., Huespe, A.E., 2015. A phase-field/gradient damage model for brittle fracture in elastic-plastic solids. Int. J. Plasticity 65, 269-296.

Gangloff, R.P., 2003. Hydrogen-assisted cracking in high-strength alloys. Comprehensive structural integrity. Environmentally-Assisted Fract. 6.

Gao, Y.F., Zhou, M., 2013. Coupled mechano-diffusional driving forces for fracture in electrode materials. J. Power Sources 230 , 176-193.

Gerberich, W.W., Chen, Y.T., 1975. Hydrogen-controlled cracking. An approach to threshold stress intensity. Metall. Trans. A 6 (2), 271. 
Germain, P., 1973. The method of virtual power in continuum mechanics. part 2: microstructure. SIAM J. Appl. Math. 25 (3), $556-575$.

Gurtin, M.E., Anand, L., 2009. Thermodynamics applied to gradient theories involving the accumulated plastic strain: the theories of aifantis and fleck and hutchinson and their generalization. J. Mech. Phys. Solids 57 (3), 405-421.

Gurtin, M.E., Fried, E., Anand, L., 2010. The Mechanics and Thermodynamics of Continua. Cambridge University Press.

Hirose, Y., Mura, T., 1984a. Nucleation mechanism of stress corrosion cracking from notches. Eng. Fract. Mech. 19 (2), $317-329$.

Hirose, Y., Mura, T., 1984b. Growth mechanism of stress corrosion cracking in high strength steel. Eng. Fract. Mech. 19 (6), $1057-1067$.

Hirth, J.P., 1980. Effects of hydrogen on the properties of iron and steel. Metall. Trans. A 11 (6), 861-890.

Jemblie, L., Olden, V., Akselsen, O.M., 20 April 2017. A coupled diffusion and cohesive zone modelling approach for numerically assessing hydrogen embrittlement of steel structures. Int. J. Hydrogen Energy 42 (16), 11980-11995.

Job, G., Herrmann, F., 2006. Chemical potential - a quantity in search of recognition. Eur. J. Phys. 27 (2), 353.

Klinsmann, M., Rosato, D., Kamlah, M., McMeeking, R.M., 2016. Modeling crack growth during Li insertion in storage particles using a fracture phase field approach. J. Mech. Phys. Solids 92, 313-344.

Larché, F.C., Cahn, J., 1982. The effect of self-stress on diffusion in solids. Acta Metall. 30 (10), 1835-1845.

Larché, F.C., Cahn, J.W., 1985. Overview nbr. 41. The interactions of composition and stress in crystalline solids. Acta metall. 33 (3), $331-357$.

Larché, F.C., Voorhees, P.W., 1996. Diffusion and stresses: basic thermodynamics. In: Defect and Diffusion Forum, vol 129. Trans Tech Publ, pp. 31-36.

Lufrano, J., Sofronis, P., Symons, D., 1998. Hydrogen transport and large strain elastoplasticity near a notch in alloy x-750. Eng. Fract. Mech. 59 (6), 827-845.

Miehe, C., Hofacker, M., Welschinger, F., 2010. A phase field model for rate-independent crack propagation: robust algorithmic implementation based on operator splits. Comput. Methods Appl. Mech. Eng. 199 (45), 2765-2778.

Miehe, C., Schänzel, L.M., Ulmer, H., 2015. Phase field modeling of fracture in multi-physics problems. part i. balance of crack surface and failure criteria for brittle crack propagation in thermo-elastic solids. Comput. Methods Appl. Mech. Eng. 294, 449-485.

Miehe, C., Aldakheel, F., Raina, A., 2016. Phase field modeling of ductile fracture at finite strains: a variational gradient-extended plasticity-damage theory. Int. J. Plasticity 84, 1-32.

Oriani, R.A., Josephic, P.H., 1974. Equilibrium aspects of hydrogen-induced cracking of steels. Acta Metall. 22 (9), $1065-1074$.

Podio-Guidugli, P., 2009. A virtual power format for thermomechanics. Continuum Mech. Thermodyn. 20 (8), $479-487$.

Serebrinsky, S., Carter, E.A., Ortiz, M., 2004. A quantum-mechanically informed continuum model of hydrogen embrittlement. J. Mech. Phys. Solids 52 (10), 2403-2430.

Simmons, G.W., Pao, P.S., Wei, R.P., 1978. Fracture mechanics and surface chemistry studies of subcritical crack growth in aisi 4340 steel. Metall. Trans. A 9 (8), $1147-1158$.

Simo, J.C., Hughes, T.J., 1998. Computational Inelasticity. Springer New York, New York, NY.

Troiano, A.R., 2016. The role of hydrogen and other interstitials in the mechanical behavior of metals. Metallogr. Microstruct. Analysis 5 (6), 557-569.

Truesdell, C.A., 1992. A First Course in Rational Continuum Mechanics, vol. 1 Academic Press.

Tvergaard, V., Hutchinson, J.W., 1992. The relation between crack growth resistance and fracture process parameters in elasto-plastic solids. J. Mech. Phys. Solids 40, 1377-1397.

Unger, D.J., 1989. A mathematical analysis for impending hydrogen assisted crack propagation. Eng. Fract. Mech. 34 (3), $657-667$.

Wu, T., De Lorenzis, L., 2016. A phase-field approach to fracture coupled with diffusion. Comput. Methods Appl. Mech. Eng. 312, $196-223$.

Xu, R., Zhao, K., 2016. Electrochemomechanics of electrodes in li-ion batteries: a review. J. Electrochem. Energy Convers. Storage 13(3) (030803).

Yu, H., Olsen, J.S., Alvaro, A., Olden, V., He, J., Zhang, Z., 2016. A uniform hydrogen degradation law for high strength steels. Eng. Fract. Mech. 157, 56-71.

Zhao, K., Pharr, M., Vlassak, J.J., Suo, Z., 2011. Inelastic hosts as electrodes for high-capacity lithium-ion batteries. J. Appl. Phys. 109 (016110). 